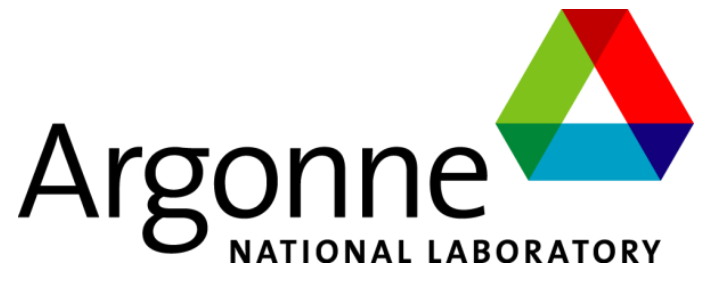

ANL-19/26

\title{
Modeling of Water Film Formation on a Stay-Cable
}

Nuclear Science and Engineering Division 


\section{About Argonne National Laboratory}

Argonne is a U.S. Department of Energy laboratory managed by UChicago Argonne, LLC under contract DE-AC02-06CH11357. The Laboratory's main facility is outside Chicago, at 9700 South Cass Avenue, Argonne, Illinois 60439. For information about Argonne and its pioneering science and technology programs, see www.anl.gov.

\section{DOCUMENT AVAILABILITY}

Online Access: U.S. Department of Energy (DOE) reports produced after 1991 and a growing number of pre-1991 documents are available free at OSTI.GOV

(http://www.osti.gov/), a service of the U.S. Dept. of Energy's Office of Scientific and

Technical Information

Reports not in digital format may be purchased by the public from the National Technical Information Service (NTIS):

U.S. Department of Commerce

National Technical Information Service

5301 Shawnee Rd

Alexandra, VA 22312

www.ntis.gov

Phone: (800) 553-NTIS (6847) or (703) 605-6000

Fax: (703) 605-6900

Email: orders@ntis.gov

\section{Reports not in digital format are available to DOE and DOE contractors from the} Office of Scientific and Technical Information (OSTI):

U.S. Department of Energy

Office of Scientific and Technical Information

P.O. Box 62

Oak Ridge, TN 37831-0062

www.osti.gov

Phone: (865) 576-8401

Fax: (865) 576-5728

Email: reports@osti.gov

\section{Disclaimer}

This report was prepared as an account of work sponsored by an agency of the United States Government. Neither the United States Government nor any agency thereof, nor UChicago Argonne, LLC, nor any of their employees or officers, makes any warranty, express or implied, or assumes any legal liability or responsibility for the accuracy, completeness, or usefulness of any information, apparatus, product, or process disclosed, or represents that its use would not infringe privately owned rights. Reference herein to any specific commercial product, process, or service by trade name, trademark, manufacturer, or otherwise, does not necessarily constitute or imply its endorsement, recommendation, or favoring by the United States Government or any agency thereof. The views and opinions of document authors expressed herein do not necessarily state or reflect those of the United States Government or any agency thereof, Argonne National Laboratory, or UChicago Argonne, LLC. 


\section{Modeling of Water Film Formation on a Stay-Cable}

prepared by

N. Sinha, M.A. Sitek, and S.A. Lottes

Nuclear Science and Engineering Division, Argonne National Laboratory

August 2019 


\section{Table of Contents}

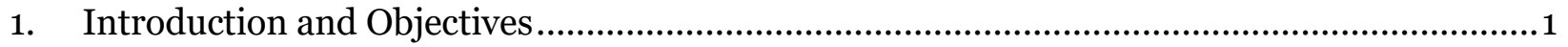

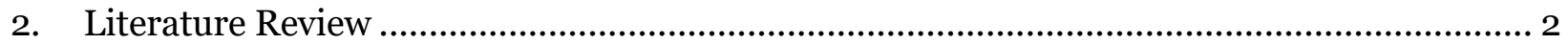

3. Governing Equations of the Computational Model .......................................................... 3

3.1. Reynolds-Averaged Navier-Stokes (RANS) Model...................................................... 3

3.2. Large Eddy Simulation (LES) Model ........................................................................ 4

3.3. Eulerian Multiphase with Volume of Fluid (VOF) Model ........................................... 5

4. Investigation of the Available Rain Modeling Methods .................................................... 6

4.1. Mass Inflow Boundary Condition on Cable without Fillets............................................7

4.2. Mass Inflow Boundary Condition on Cable with Fillets .............................................10

4.3. Lagrangian Transport Model ......................................................................................12

4.4. Mass Source Term ….......................................................................................... 15

4.4.1. $\quad 30 \mathrm{~mm} / \mathrm{hr}$ Rain Intensity .......................................................................... 17

4.4.2. $\quad 60 \mathrm{~mm} / \mathrm{hr}$ Rain Intensity .......................................................................... 19

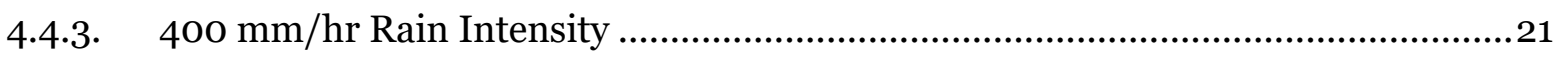

5. Influence of Crosswind Flow on Rivulet Formation and Flow......................................... 22

5.1. Instantaneous and Mean Flow Field in Dry Conditions............................................... 22

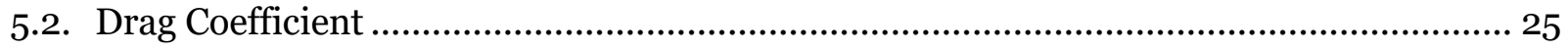

5.3. Film Thickness and Upper Rivulet Formation ....................................................... 26

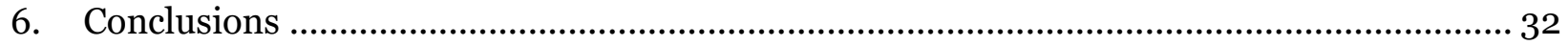

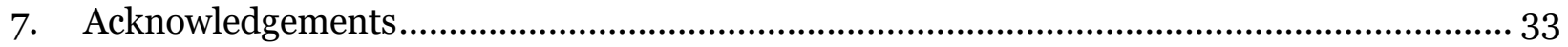

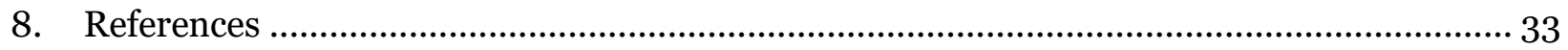




\section{List of Figures}

Figure 1-1 The Cooper River Bridge (Arthur Ravenel Jr. Bridge), one of North America's longest cable-stayed bridges connects Charleston to Mount Pleasant, SC.

Figure 1-2 Bridge cable with two helical fillets 1

Figure 4-1 General view of the domain with a bridge cable. The cable is positioned at an angle alpha to the horizontal plane XY. 8

Figure 4-2 Volumetric mesh on a cross-sectional vertical plane (XZ) going through the cable .... 8

Figure 4-3 Initial film thickness with the upper side of cable covered with a $1 \mathrm{~mm}$ film (initial wet surface assumption). The color scale is: $\mathrm{red}=100 \%$ water and blue $=100 \%$ air. The location of the inflow boundary condition surface is shown above the top edge of the top surface........... 9

Figure 4-4 Film formation on lower (left) and upper (right) side of the cable ......................... 9

Figure 4-5 Fluid film on the cable. The vertical axis denotes the length along the axis of the cylinder while the bottom axis denotes the angle along the circumference of the cylinder with angle 270 degrees on the top of the cable.

Figure 4-6 Snapshots showing mesh resolution on a cross-sectional plane and on the cable surface with fillets.

Figure 4-7 Film formation on the bottom (left) and the top (right) side of the cable 11

Figure 4-8 Rivulet and film thickness on cable with fillets. The vertical axis denotes the length along the axis of the cylinder while the bottom axis denotes the angle along the circumference of the cylinder with angle 270 degrees at the top of the cable......

Figure 4-9 Injection of the Lagrangian particles into the domain, simulating rainfall. 13

Figure 4-10 Fluid film thickness on the cable with fillets. The vertical axis denotes the length along the axis of the cylinder while the bottom axis denotes the angle along the circumference of the cylinder with angle 270 degrees at the top of the cable.

Figure 4-11 Velocity of water in the fluid film on the cable surface.........................................14

Figure 4-12 Computational domain used in this part of the study .........................................15

Figure 4-13: Mesh description for LES simulations ..........................................................16

Figure 4-14 Location of the mass source term region for rain shown in blue.............................16

Figure 4-15 Rainfall intensity vs duration curves for various events in Louisiana. Source: http://hdsc.nws.noaa.gov/hdsc/pfds/ 
Figure 4-16 Fluid film thickness on the cable for $30 \mathrm{~mm} / \mathrm{hr}(1.2 \mathrm{in} / \mathrm{hr})$ rain. The vertical axis denotes the length along the axis of the cylinder while the bottom axis denotes the angle along the circumference of the cylinder with angle 270 degrees at the top of the cable.....

Figure 4-17 Rivulets on the bottom (left) and top (right) side of the cable

Figure 4-18 Fluid film thickness on the cable for $60 \mathrm{~mm} / \mathrm{hr}(2.4 \mathrm{in} / \mathrm{hr})$ rain intensity. The vertical axis denotes the length along the axis of the cylinder while the bottom axis denotes the angle along the circumference of the cylinder with angle 270 degrees at the top of the cable...20

Figure 4-19: Rivulet formation on the bottom (left) and top (right) side of the cable. 20

Figure 4-20 Fluid film thickness on the cable for $400 \mathrm{~mm} / \mathrm{hr}$ rain. The vertical axis denotes the length along the axis of the cylinder while the bottom axis denotes the angle along the circumference of the cylinder with angle 270 degrees at the top of the cable.....

Figure 4-21: Rivulet formation on the bottom (left) and the top (right) end of the cable........... 22

Figure 5-1 Vorticity magnitude for crosswind flow over cable in dry conditions, (a) plain cylinder and (b) cylinder with filllet

Figure 5-2 Coherent structures based on q-criteria with no fillets (left) and with fillets (right) . 23

Figure 5-3 Shear stress distribution at wind velocity $6.2 \mathrm{~m} / \mathrm{s}$ on the surface of a (a) plain cylinder, and (b) cylinder with fillets. The vertical axis is distance along the axis of the cylinder while the bottom axis is the angle along the circumference of the cylinder with angle o degrees on the leeward side of the cable.

Figure 5-4 Contours of normalized downstream velocity (non-positive on a cross section plane for (a) plain cylinder, (b) cylinder with fillet (c) wet cylinder with fillet....

Figure 5-5 Time history of the drag coefficient, Cd, for cylinders: without fillets (black), with fillets (blue), and with fillets and water film (red).

Figure 5-6 Wind velocity $5.0 \mathrm{~m} / \mathrm{s}$ and $60 \mathrm{~mm} / \mathrm{hr}(2.4 \mathrm{in} / \mathrm{hr})$ rain: fluid film thickness on the cable surface (left), and film velocity (right). The vertical axis is the length along the axis of the cylinder while the bottom axis is the angle along the circumference of the cylinder with angle 270 deg at the top of the cable.

Figure 5-7 Wind velocity $6.2 \mathrm{~m} / \mathrm{s}$ and $60 \mathrm{~mm} / \mathrm{hr}(2.4 \mathrm{in} / \mathrm{hr})$ rain: fluid film thickness on the cable surface (left), and film velocity (right). The vertical axis is the length along the axis of the cylinder while the bottom axis is the angle along the circumference of the cylinder with angle 270 deg at the top of the cable.

Figure 5-8 Wind velocity $7.2 \mathrm{~m} / \mathrm{s}$ and $60 \mathrm{~mm} / \mathrm{hr}(2.4 \mathrm{in} / \mathrm{hr})$ rain: fluid film thickness on the cable surface (left), and film velocity (right). The vertical axis is the length along the axis of the cylinder while the bottom axis is the angle along the circumference of the cylinder with angle $270 \mathrm{deg}$ at the top of the cable. 
Figure 5-9 Wind velocity $7.7 \mathrm{~m} / \mathrm{s}$ and $60 \mathrm{~mm} / \mathrm{hr}(2.4 \mathrm{in} / \mathrm{hr})$ rain: fluid film thickness on the cable surface (left), and film velocity (right). The vertical axis is the length along the axis of the cylinder while the bottom axis is the angle along the circumference of the cylinder with angle $270 \mathrm{deg}$ at the top of the cable.

Figure 5-10 Wind velocity $8.7 \mathrm{~m} / \mathrm{s}$ and $60 \mathrm{~mm} / \mathrm{hr}(2.4 \mathrm{in} / \mathrm{hr})$ rain: fluid film thickness on the cable surface (left), and film velocity (right). The vertical axis is the length along the axis of the cylinder while the bottom axis is the angle along the circumference of the cylinder with angle 270 deg at the top of the cable. 


\section{Introduction and Objectives}

With the increasing popularity of cable-stayed bridges in the past few decades, longer spans are being constructed using ever-longer stay cables. Under certain conditions, especially during construction, and wind and rain-wind events, the cables of cable-stayed bridges may undergo large amplitude vibrations. This type of vibration has been of concern to researchers and engineers because large amplitude vibration can induce excessive stresses and therefore threaten the safety and integrity of bridge structures. Figure 1-1 presents an example of a stay cable bridge structure, The Cooper River Bridge. Many countermeasures for the wind-rain induced vibrations have been developed to decrease the amplitude of the vibrations and consequently, the fatigue of the cables. One of the methods is to install helical fillets on the cable surface. A sketch of a cable with two fillets wrapped around it is shown in Figure 1-2.

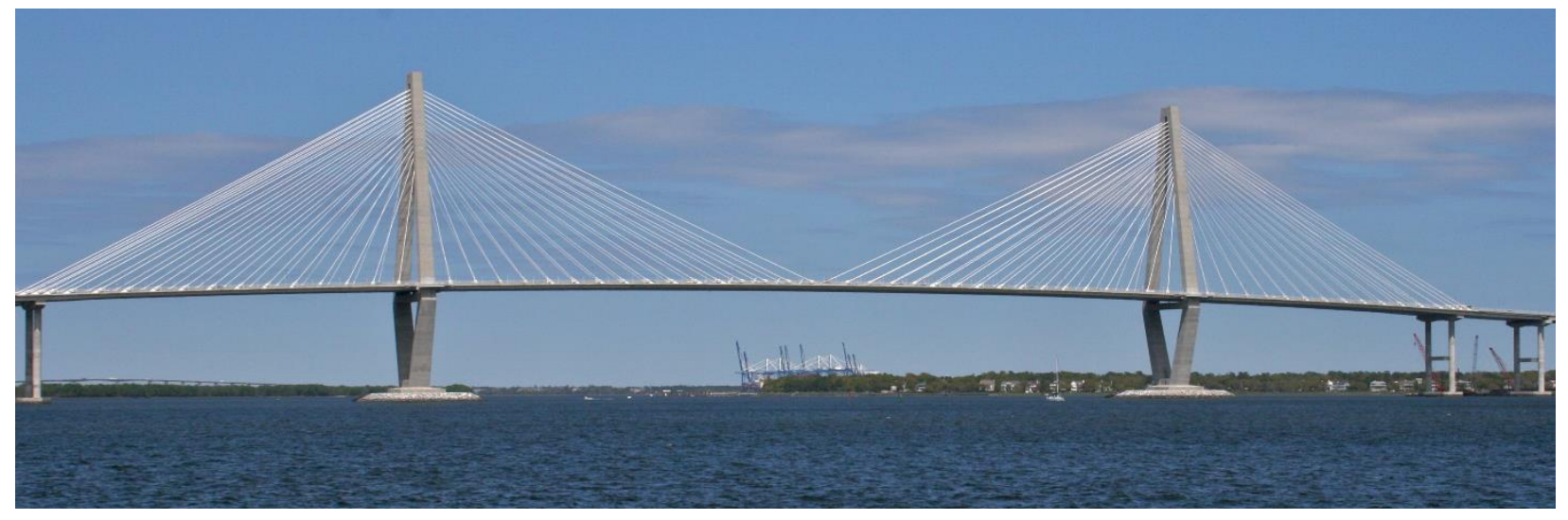

Figure 1-1 The Cooper River Bridge (Arthur Ravenel Jr. Bridge), one of North America's longest cable-stayed bridges connects Charleston to Mount Pleasant, SC.

(by : bbatsell under Creative Commons Attribution-Share Alike 2.5 Generic license)

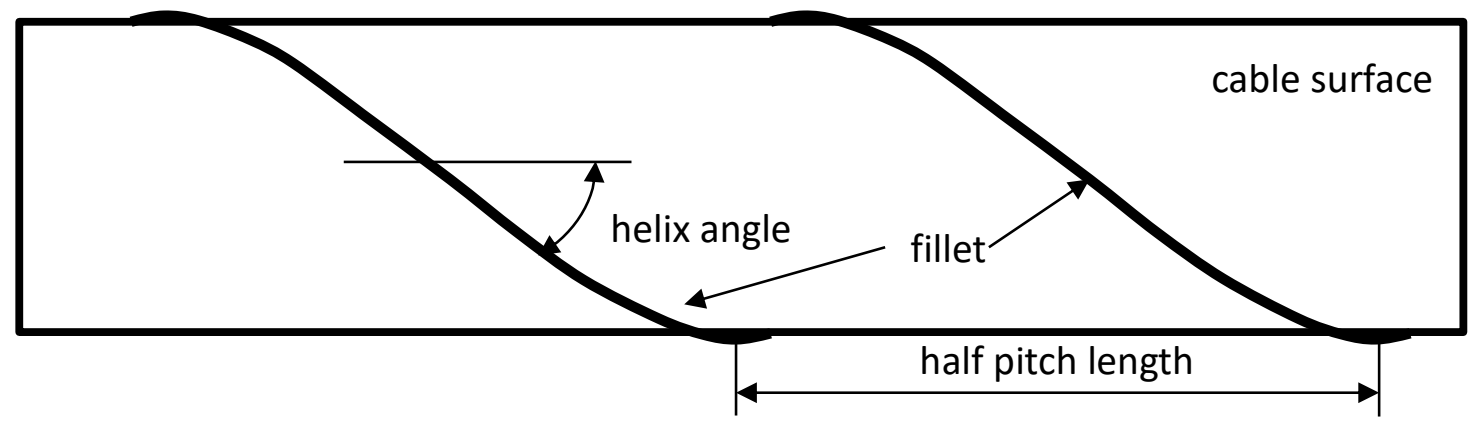

Figure 1-2 Bridge cable with two helical fillets 
The main objective of the present study is to develop and test computational methodologies, with the use of computational fluid dynamics (CFD), that model the interaction of rain with stay cables including: the formation of water film, development of rainwater rivulets, and rivulet behavior under various conditions with and without wind. Interaction of water with cables with and without fillets is analyzed, and results are presented in this report.

This study is divided into two parts. The first part of this study, Section 4, explores methods of modeling water film formation and flow on cables. A series of simulations using the Reynolds averaged Navier-Stokes equations turbulence model (RANS) and large eddy simulation (LES) model are performed to analyze water film formation and flow on a cable using the fluid film model in STARCCM+ [(b)1]. Various methods of setting up initial and boundary conditions are tested, especially boundary conditions for introducing rain into the domain. The second part of the study, Section 5, explores upper rivulet formation in the presence of wind, based on the findings from the first part of the study. In that section, LES simulation with the Volume Of Fluid model for the free surface is used. Also, various results for cases with and without water film on the cable are presented and compared.

\section{Literature Review}

Wind induced cable vibrations in cable-stayed bridges were first observed in Japan [2] during the construction of the Meikonishi bridge. The engineers were the first to note rain-wind induced vibration and claim that the vibrations were neither due to wake galloping nor to vortex-induced vibration. They performed full-scale measurements and confirmed that the frequency of the observed vibrations was well below the critical frequency of the vortex-induced vibration. They also ruled out the possibility that the observed vibration was a consequence of wake galloping as the distances between the cables were not large enough to cause interference with each other.

Hikami and Shirashi [2] also made important observations about the formation of upper and lower rivulets on a cable. They reported that upper rivulets play an important role in the occurrence of vibrations and can only form within specific ranges of rain and wind velocity conditions. Further, Matsumoto et al. [3] reported that upper rivulets affect the three dimensionalities of the Karman vortex shedding, resulting in rain-vortex induced vibration.

There are two ways for mimicking rivulets on the stay cable in wind tunnel tests. First is to place artificial rivulets on the stay cable [4], while second is to spray water onto the surface of the stay cable to form rivulets. Kleissl \& Georgakis [5] performed several tests using water spray on various cables with surface modifications to predict rivulet formation and suppression.

Computational solutions of the rivulet formation problem are scarce. Chen et al. [6] and Li and $\mathrm{Gu}$ [7] reported 2D CFD simulations to study the influence of dynamic properties and position of the upper rivulet on rain-wind induced vibration of stay cables. They used the artificial rivulet method (a convex hump fixed on the cylinder) in the simulation. A 2D and 3D analysis using LES in a commercial code, Fluent [8], showed the importance of grid refinement in the radial direction to obtain an accurate vortex shedding frequency. Further, the simulations showed that the aerodynamic behaviour of the $3 \mathrm{D}$ model was different from that of the $2 \mathrm{D}$ model. Vortex shedding 
in the case of a $3 \mathrm{D}$ cylinder was irregular and delayed the boundary layer separation of the approach flow compared to 2D simulation.

A numerical simulation of the formation of rivulets on a stay-cable resolving the gas-liquid interface with the use of the volume of fluid (VOF) method was presented by Bi et al [9]. The effect of crosswind and gravity on the morphology of rivulets was analyzed in the paper. The study verifies the accuracy and reliability of using the VOF method to simulate the morphology of a liquid film on the cable surface. The authors conclude that under a combination of gravity and wind, the location of upper rivulets moves downward and thickness of lower rivulets increases as compared to the flow without either wind or gravity, which was consistent with the experimental results. The VOF method was also used by Bi et al [10] to determine the aerodynamic forces when rain-wind induced vibration occurs. The vibration responses were close to experimental results. It was confirmed that when the upper rivulets formed at a specific wind speed, they could lead to a periodic change in aerodynamic lift, which in turn would produce rain-wind induced vibrations.

Overall, the literature review showed that the rain-wind induced vibration only happens under certain conditions, i.e. mild wind and rain, and that it is sensitive to the orientation of the stay cable. Moreover, the upper and lower rivulets, could affect the period of the aerodynamic lift oscillation, with the upper rivulet being one of the important factors causing rain-wind induced vibrations.

\section{Governing Equations of the Computational Model}

The simulations are performed using STAR-CCM+ computational fluid dynamics (CFD) software. For the problem at hand, a set of flow and turbulence modeling are used. Both the ReynoldsAveraged Navier-Stokes (RANS) turbulence model and the Large Eddy Simulation (LES) were used for parts of the study.

\subsection{Reynolds-Averaged Navier-Stokes (RANS) Model}

The Reynolds-Averaged Navier-Stokes (RANS) equations can be obtained by decomposing the instantaneous velocity and pressure fields of Navier-Stokes equations into a mean value and a fluctuating component, averaging the equations in terms of those variables, and then adding model terms for cross correlations. The unsteady form of the governing Reynolds Averaged Navier Stokes (URANS) equations is given in the STAR-CCM+ User Guide [1] in terms of averaged variables, $\bar{\phi}$, where $\phi=\bar{\phi}+\phi^{\prime}$ is the variable from the unaveraged governing continuum equations, and $\phi^{\prime}$ is the turbulent fluctuating component of $\phi$.

Conservation of mass:

$$
\frac{\partial \rho}{\partial t}+\nabla \cdot[\rho(\bar{v})]=0
$$

Conservation of momentum, Newton's 2nd law for the fluid motion: 


$$
\frac{\partial}{\partial t}(\rho \overline{\boldsymbol{v}})+\nabla \cdot[\rho \bar{v} \otimes \bar{v}]=-\nabla \cdot \bar{p} \mathbf{I}+\nabla \cdot\left(\mathrm{T}+\mathrm{T}_{\mathrm{RANS}}\right)+f_{b}
$$

where:

$$
\begin{array}{clcl}
\rho & \text { density } & t & \text { time } \\
f_{b} & \text { body force (gravity) } & \bar{p} & \text { mean pressure } \\
\mathbf{T} & \text { viscous stress tensor } & \mathbf{I} & \text { identity matrix } \\
\bar{v} & \text { mean velocity } & \mathbf{T}_{R A N S} & \text { Reynolds stress tensor }
\end{array}
$$

The Realizable Two-Layer k- $\varepsilon$ turbulence model $[11,12]$ was used with the all y+ wall treatment since this model works well for Reynolds stress tensors when there are varying mesh densities in the domain. In this eddy viscosity turbulence model

$$
\mathbf{T}_{R A N S}=2 \mu_{t} \mathbf{S}-\frac{2}{3}\left(\mu_{t} \nabla \cdot \overline{\boldsymbol{v}}\right) \mathbf{I}
$$

where $\mu_{t}$ is the eddy viscosity and $\mathbf{S}=\left(\nabla \overline{\boldsymbol{v}}+\nabla \overline{\boldsymbol{v}}^{T} \overline{)} / 2\right.$ is averaged strain rate tensor. The all y+ wall treatment is a composite model for obtaining the wall shear stress under conditions where the non-dimensional distance from the wall to the first computational grid point, known as $y+$, may be outside the range of 30 to 100 suitable for application of standard wall functions on some areas of the wall boundary. Details are available in the STAR-CCM+ user guide [1].

\subsection{Large Eddy Simulation (LES) Model}

Large eddy simulation is an inherently transient turbulence modeling technique in which the large scales of turbulence are resolved in the flow domain, and the small-scale turbulent motions are modeled. In the LES model governing equations, the variables, $\phi$ are split into a filtered value $\tilde{\phi}$ and a sub-grid value $\phi^{\prime}$ with $\phi=\tilde{\phi}+\phi^{\prime}$. Sub-grid values are modeled.

Conservation of mass:

$$
\frac{\partial \rho}{\partial t}+\nabla \cdot[\rho \tilde{v}]=0
$$

Conservation of momentum, Newton's 2nd law for the fluid motion:

$$
\frac{\partial}{\partial t}(\rho \tilde{v})+\nabla \cdot[\rho \tilde{v} \otimes \tilde{v}]=-\nabla \cdot \tilde{\mathrm{p}} \mathbf{I}+\nabla \cdot\left(\mathrm{T}+\mathrm{T}_{\mathrm{SGS}}\right)+f_{b}
$$

where:

$\rho$ density $t$ time 
$f_{b} \quad$ body force (gravity)

$\tilde{v} \quad$ filtered velocity

$\mathrm{T} \quad$ viscous stress tensor $\tilde{\mathrm{p}} \quad$ filtered pressure

I identity matrix

$\mathrm{T}_{\mathrm{SGS}} \quad \mathrm{SGS}$ stress tensor

and

$$
\mathbf{T}_{\boldsymbol{S G S}}=2 \mu_{t} \mathbf{S}-\frac{2}{3}\left(\mu_{t} \nabla \cdot \widetilde{\boldsymbol{v}}\right) \mathbf{I}
$$

Here, $\mathbf{S}$ is the strain rate tensor computed using equation 3 with the filtered velocity $\widetilde{\boldsymbol{v}}$ in place of $\bar{v}, \mu_{t}$ is a modeled sub-grid scale eddy viscosity.

To complete the subgrid-scale (SGS) turbulence model, the wall adaptive local eddy viscosity (WALE) model [13] was used with an all $\mathrm{y}+$ wall treatment. The all $\mathrm{y}+$ wall treatment method is capable of resolving the boundary layer velocity distribution for a sufficiently fine grid, and in that case, it obtains the wall shear stress directly from the discrete form of the definition.

\subsection{Eulerian Multiphase with Volume of Fluid (VOF) Model}

To accurately simulate the free surface of the water flow on a cable section, the Eulerian Multiphase and Volume of Fluid physics models are selected in STAR-CCM+. The Volume of Fluid method can model an air-water domain with a free surface interface that divides the water zones and air zones by solving a single set of momentum and mass conservation equations and tracks the volume fraction of water in each computational cell over the entire domain.

The equations for the multiphase fluid material properties of the air-water mixture in a computational cell containing the free surface for the VOF model are:

$$
\rho=\sum_{i} \rho_{i} \alpha_{i}, \mu=\sum_{i} \mu_{i} \alpha_{i}, c_{p}=\sum_{i} \frac{\left(c_{p}\right)_{i} \rho_{i}}{\rho} \alpha_{i}
$$

where:

$$
\alpha_{i}=\frac{V_{i}}{V}
$$

is the volume fraction, $V_{i}$ is the volume occupied by the $\mathrm{i}^{\text {th }}$ phase, $V$ is the total volume, and $\rho_{i}, \mu_{i}$, and $\left(c_{p}\right)_{i}$ are the density, molecular viscosity, and specific heat of the $\mathrm{i}^{\text {th }}$ phase respectively.

The conservation equation that describes the transport of volume fraction, $\alpha_{i}$, is:

$$
\frac{d}{d t} \int_{V} \alpha_{i} d V+\int_{S} \alpha_{i}(\tilde{v}) \cdot d \boldsymbol{a}=\int_{V} S_{\alpha_{i}} d V
$$

where $S_{\alpha_{i}}$ is a source or sink term of the $i^{\text {th }}$ phase. 
For only two phases, air and water, only one phase volume equation needs to be solved because the volume fraction of the other phase is one minus the volume fraction of the phase being solved for.

In an effort to represent the rain droplets falling on a cable realistically, a fully coupled Lagrangian multiphase model was also tested. In this model, each raindrop is tracked until it adheres to the cable surface or any other surface and becomes a part of the fluid film or flows out of the domain.

A fluid film model was tested as well. This model solves the transport equations for mass, momentum, and volume fraction for a single layer of computational cells next to a wall surface containing the fluid film. It assumes that the film is thin enough to consider a laminar flow of the fluid with a parabolic velocity profile. The primary purpose of testing this model is to avoid resolving a thin layer of the fluid and therefore save on the computational resources and time. Details of this model can be found in STAR-CCM+ user guide [1].

Further, a rain impingement and a film striping model were also considered to enhance the fluid film model. The Bai-Gosman impingement model helps to compute the momentum transfer between raindrops and fluid film on the cable. The film-striping model is used to compute the fraction of liquid stripped out of the liquid film due to gravity, film acceleration, and sharp edges.

Additional details of the complete set of model governing equations, including the transport equations for the turbulent kinetic energy and dissipation rate in the k-epsilon turbulence model and the wall functions used to compute wall shear can be found in the STAR-CCM+ User Guide [1].

\section{Investigation of the Available Rain Modeling Methods}

A series of initial tests were performed to establish the most efficient method of modeling the development and behavior of water film and rivulets on a stay cable, with and without fillets, under various air flow conditions with currently available moderate sized parallel cluster computer systems. The Reynolds-Averaged Navier-Stokes (URANS) solver was used in this set of simulations. In all cases gravitational acceleration and surface tension are included in the computational model.

The computational domain is a box with dimensions of $12 \mathrm{D}$ by $7 \mathrm{D}$ by $15 \mathrm{D}$ (where $\mathrm{D}$ is the diameter of the cable) in length, width, and height respectively. A no-slip wall boundary condition was used for the cable surface, and all the boundaries of the bounding box were set to a symmetry boundary (zero gradient). In this set of simulations, the rain and crosswind were not included.

The cable was positioned in the middle of the box, at an angle to the horizontal plane. Figure 4-1 presents an isometric view of the domain with the cable inside. The domain is meshed out with a hexahedral mesh, with cells aligned with the cable. The mesh is denser close to the cable surface to capture the thin water film. The volumetric mesh on a cross-sectional vertical plane (XZ) is shown in Figure 4-2.

Water was introduced into the domain in several ways. The first approach was to model 'an artificial rivulet'. A small ellipsoidal surface was created on the top half of the top edge of the cable 
and used as an inflow boundary condition to simulate a small stream of water that would be present on the surface of the cable in the section above the area of interest. Additionally, a thin water film was applied on the cable surface at the beginning of the simulation to represent inflow of rain. Figure 4-3 illustrates the described conditions.

Secondly, Lagrangian particles were injected through a section of the top surface of the domain to mimic the inflowing rain. This method gives a very realistic representation of falling water droplets. The particles can be injected with a variety of diameters, and at a chosen flow rate. Each of the particles can represent one, or a cluster, of rain drops. This simulation was also initiated with a water film on the cable.

In the third approach the water was introduced into the domain with the use of a mass source in a volume above the top surface of the cable.

The geometry of a plain cable used in the preliminary analysis of this study was represented with a circular cylinder. A section of a bridge cable was modeled as a cylinder with double helix fillets on the surface. The model of a stay cable used in this study is similar to the one used in wind tunnel experiments by Larose and D'Auteuil [14]. The double helix fillets on the cable surface were $2.3 \mathrm{~mm}$ thick and $2.4 \mathrm{~mm}$ wide with a pitch of $520 \mathrm{~mm}$ and a helix angle of 44.4 degrees, matching the experimental model. The cable length and diameter were modified: the length was decreased from 22 feet to 5.6 feet, and the diameter is changed from 6.2 inches to 4 inches. These changes didn't inflence the results of interest, i.e. formation of the rivulets, but allowed a reduction of the computational resources and time of the simulations. The surface of the cable was considered to be smooth in the computations, as the roughness of the experimental model of the cable was about 0.6 microns, making it effectively hydraulically smooth for the purpose of determining wall friction of the flow.

\subsection{Mass Inflow Boundary Condition on Cable without Fillets}

In this computational model, the formation of rivulets on the cable surface was simulated using the fluid film model in combination with the VOF model. The two continuous phases of the flow field were air and water. The interface between the two fluids was defined as a free surface, and no mass transfer was allowed between the fluids. The reference pressure of the flow field was standard atmospheric pressure, and the acceleration of gravity was set along the vertical axis of the global coordinate system.

Initially, the upper side of cable was covered with a $1 \mathrm{~mm}$ water film, as shown in Figure 4-3. Water entered the domain throughout the simulation via a small surface located on the top half of the top edge of the cable. The mass inflow flow rate was equivalent to a $60 \mathrm{~mm} / \mathrm{hr}(2.4 \mathrm{in} / \mathrm{hr})$ rain rate. 


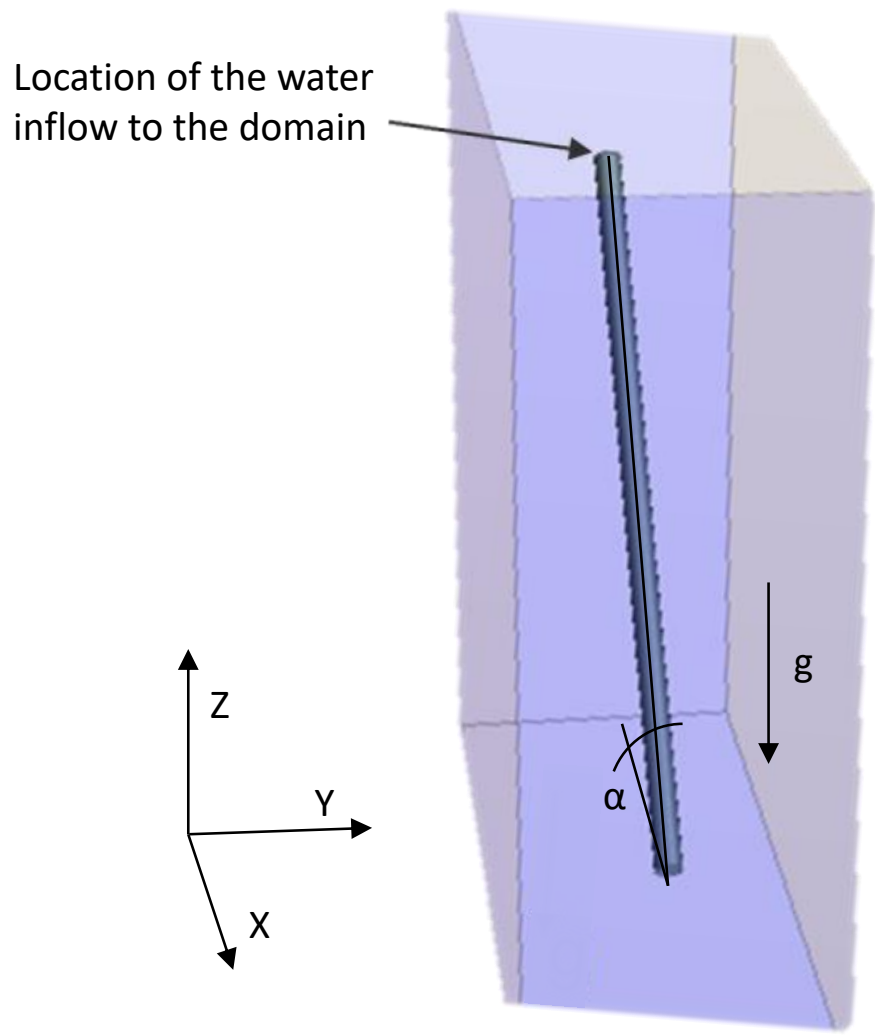

Figure 4-1 General view of the domain with a bridge cable. The cable is positioned at an angle alpha to the horizontal plane XY.

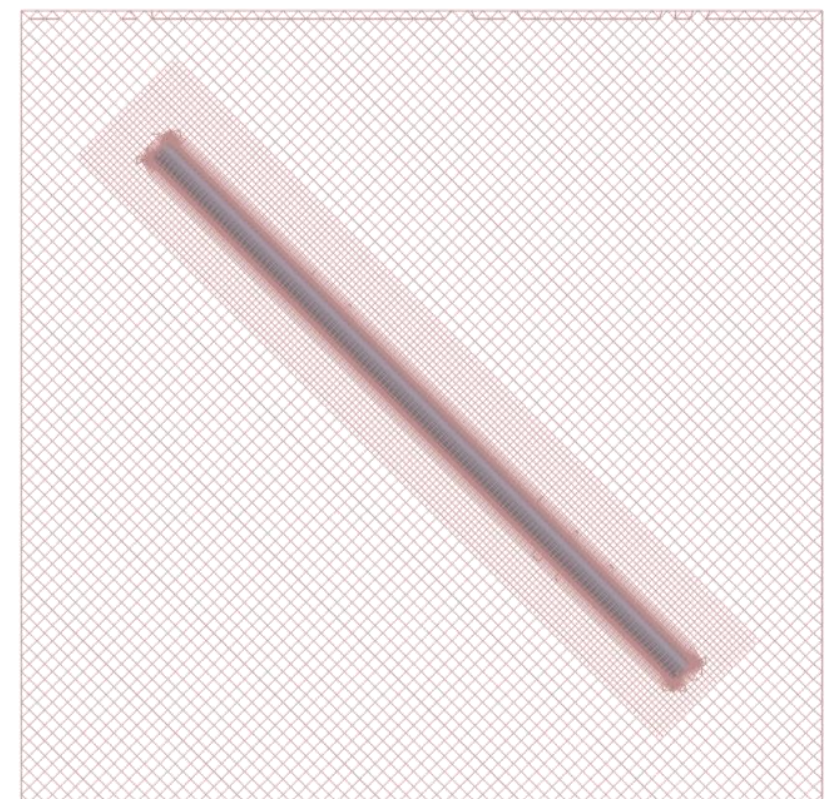

Figure 4-2 Volumetric mesh on a cross-sectional vertical plane (XZ) going through the cable 


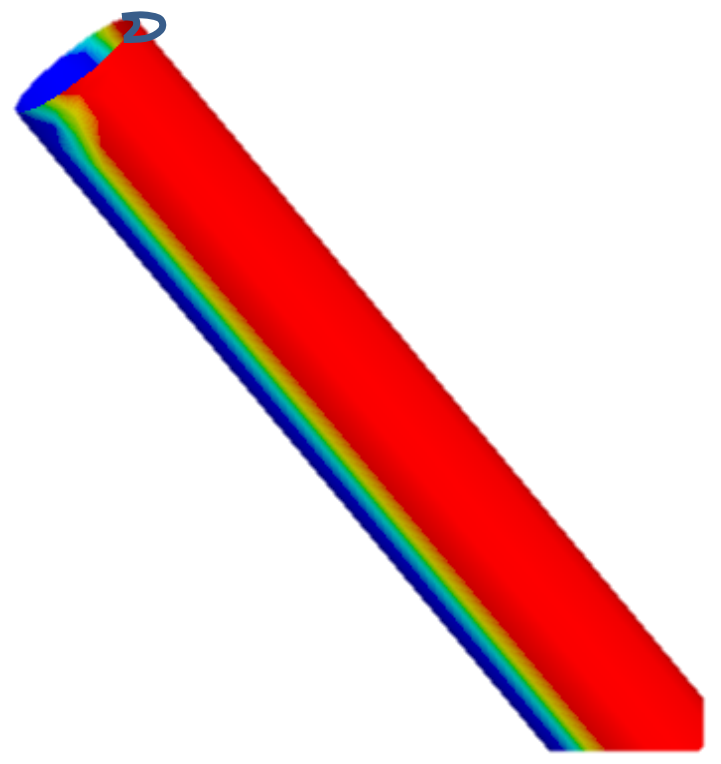

Figure 4-3 Initial film thickness with the upper side of cable covered with a $1 \mathrm{~mm}$ film (initial wet surface assumption). The color scale is: red $=100 \%$ water and blue $=100 \%$ air. The location of the inflow boundary condition surface is shown above the top edge of the top surface.

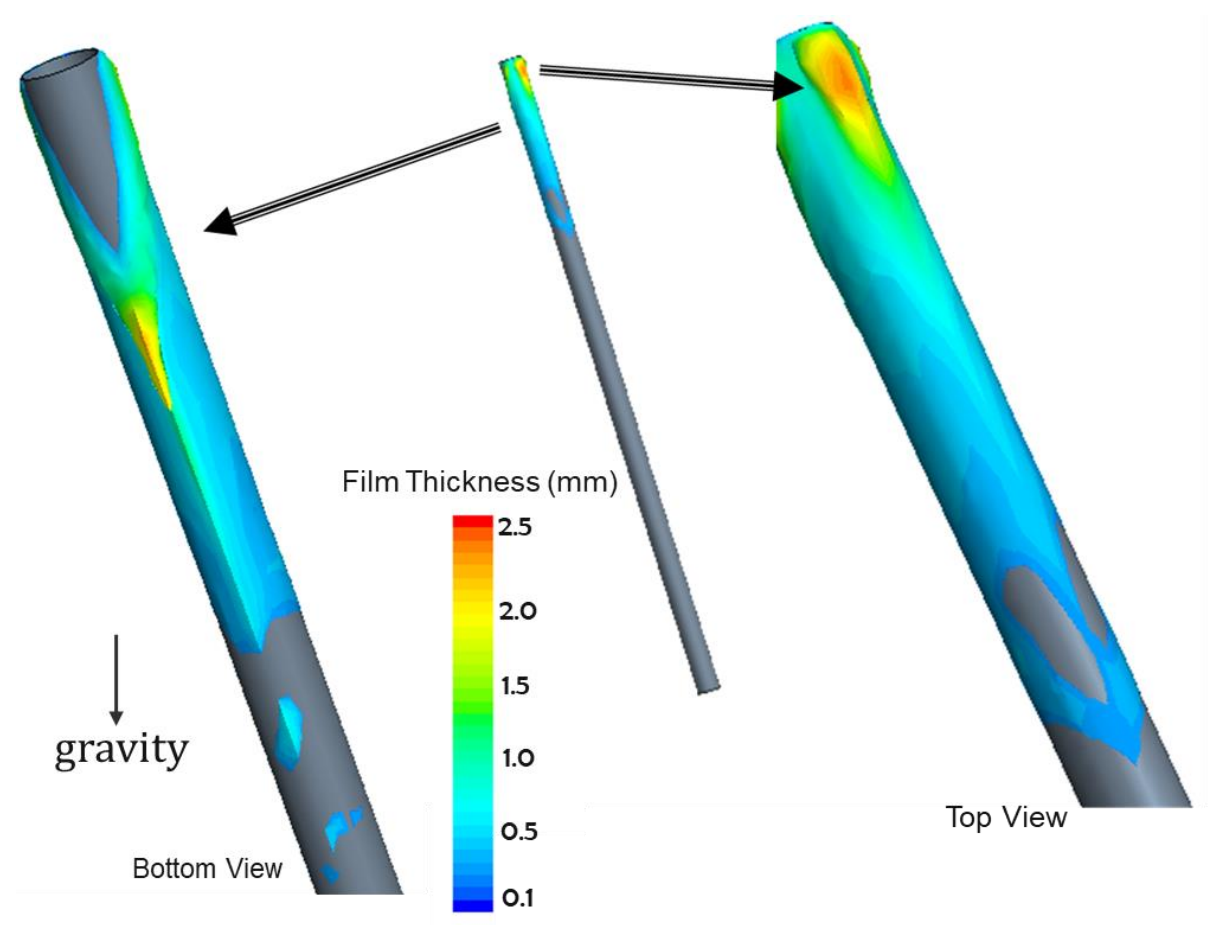

Figure 4-4 Film formation on lower (left) and upper (right) side of the cable 


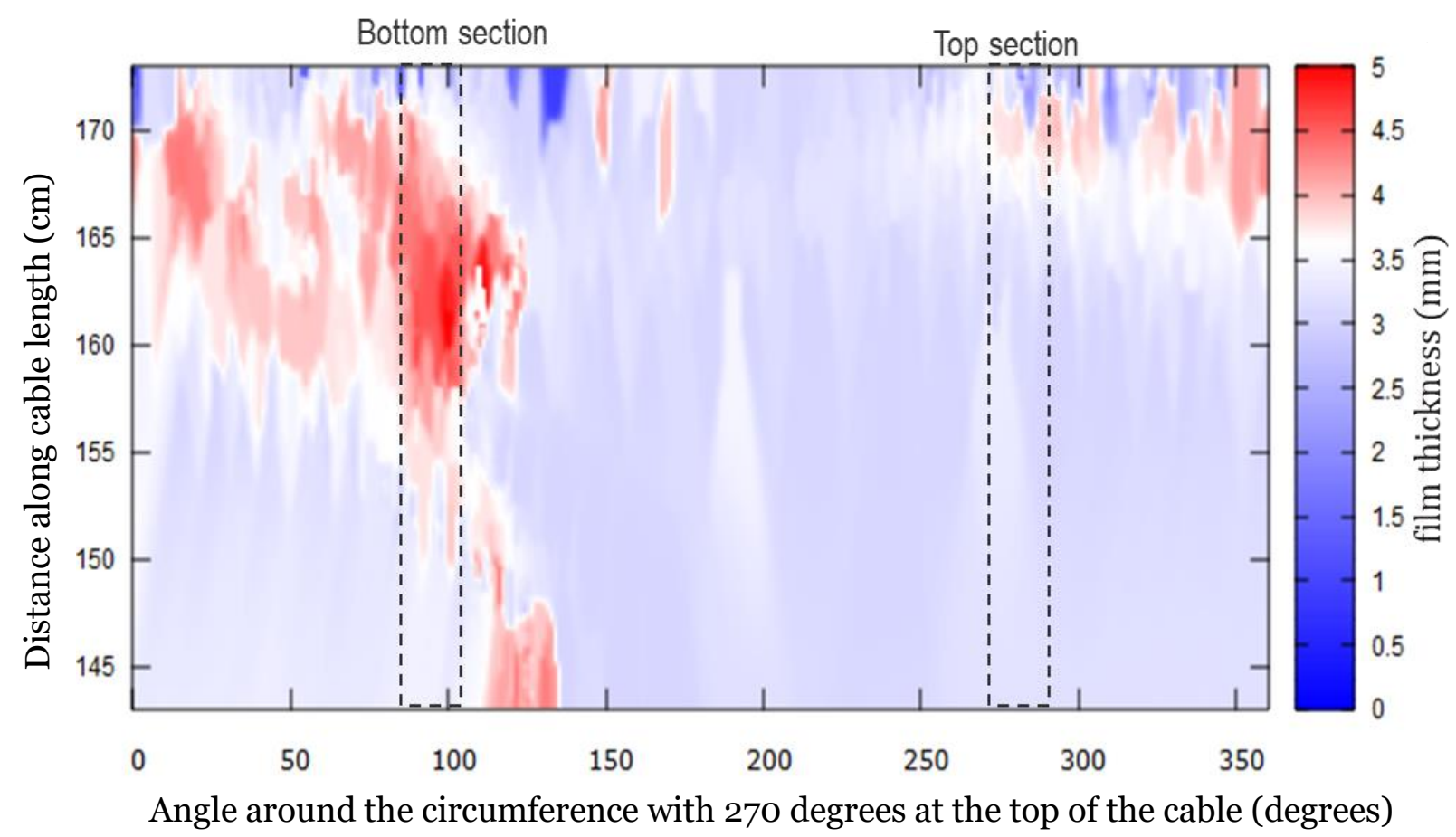

Figure 4-5 Fluid film on the cable. The vertical axis denotes the length along the axis of the cylinder while the bottom axis denotes the angle along the circumference of the cylinder with angle 270 degrees on the top of the cable.

Figure 4-4 shows and isometric view of the cable with a water film on the surface under gravitational force and with no crosswind at simulated time $t=1 \mathrm{~s}$. Figure 4-4 shows a contour plot of the water film thickness distribution on the top 30\% of the cable length. Water film formation is limited to this section, and the part of the cable surface where water film thickness is zero, was omitted. The elongated red streak represents one rivulet on the bottom section of the cable, 90 degrees from the stagnation point. The thickness of the rivulet reaches $5 \mathrm{~mm}$ and the water film thickness at all other locations on the cable surface is near to, or less than, $1 \mathrm{~mm}$ and is consistent with the prescribed initial condition.

\subsection{Mass Inflow Boundary Condition on Cable with Fillets}

The effect of surface irregularity, due to the presence of fillets, on water film formation was analyzed next. The fillets used in this simulation were a double helix type with $2.4 \mathrm{~mm}$ thickness. The VOF model was used to resolve the film behaviour on the cable. Water flow into the domain through a small surface at the top edge of the cable, mimicking the upstream water flow on the cable, as described in the chapter 4.1. The computational domain, initial conditions, and boundary conditions were the same as the previous case with no fillets. For the purpose of producing a smooth grid, a trimmed grid model with fine cells near cylinder was used. The total volume mesh count for this domain was $\sim 3$ million, with a near cylinder cell size of $\sim 100$ microns. Some mesh details are presented in Figure 4-6. 

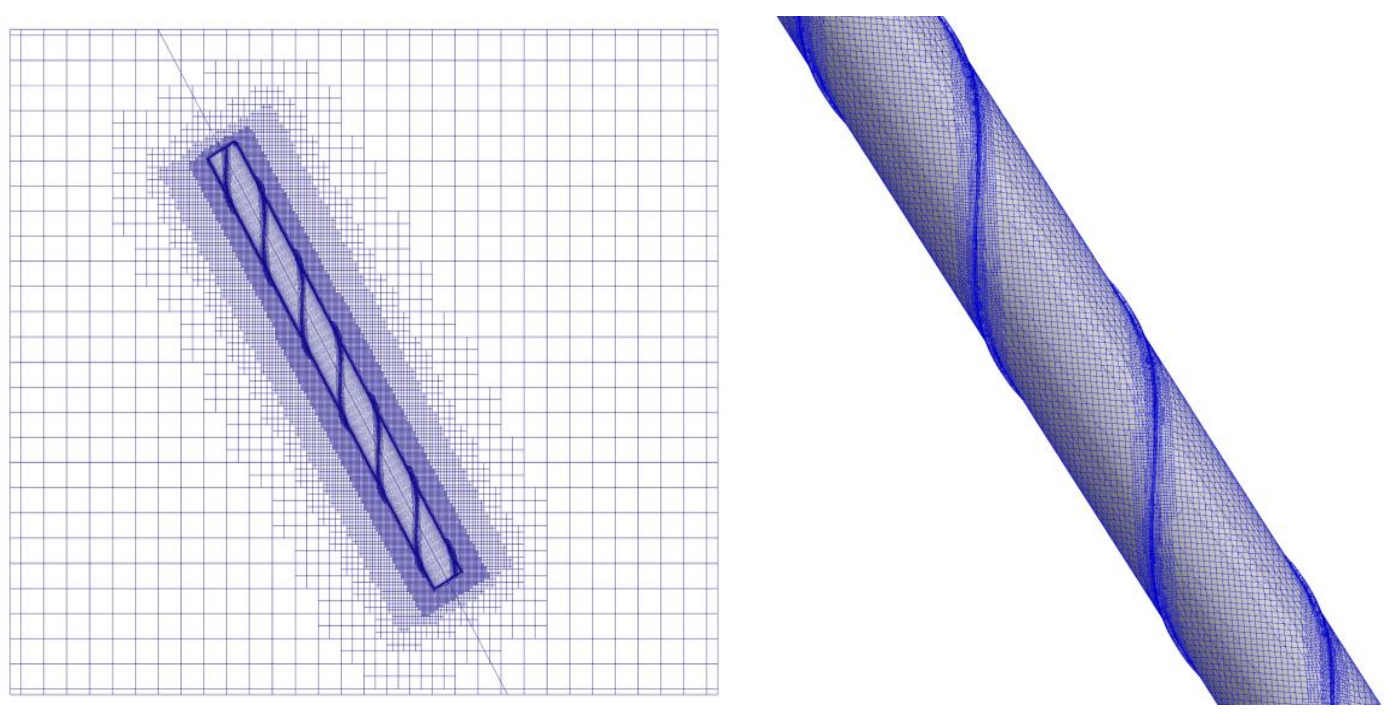

Figure 4-6 Snapshots showing mesh resolution on a cross-sectional plane and on the cable surface with fillets

Figure 4-7 and Figure 4-8 show the water film on the upper 90\% of the cable length with no crosswind at time $t=\sim 1 \mathrm{~s}$ under gravity and with inflow from the top. The upper $30 \%$ of the cable is covered with a thick water film. The water, injected on the top of the cable slides along the fillets until it overtops them and strips away from the cable due to gravity. Two distinct streaks of thickness $\sim 1.25 \mathrm{~mm}$ formed at 90 degrees (lower side) and 270 degrees (upper side) of the cable. The formation of two streaks is consistent with experimental and numerical results [10].
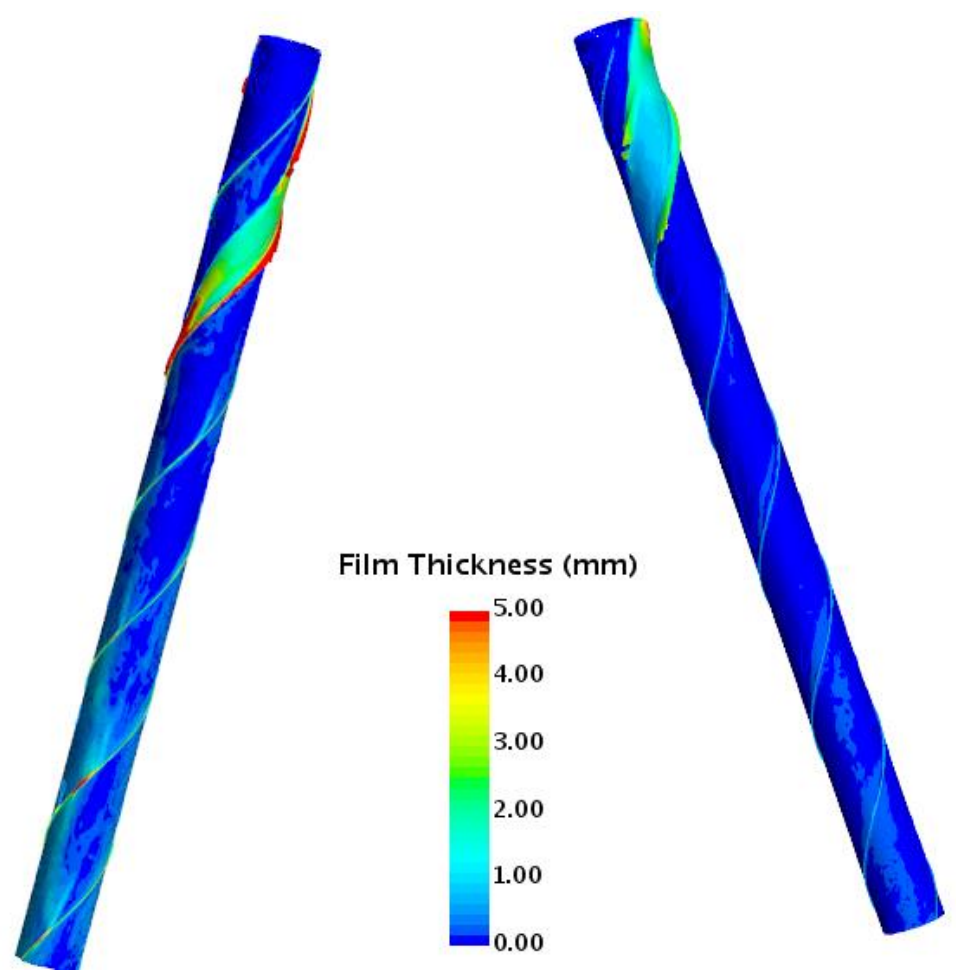

Figure 4-7 Film formation on the bottom (left) and the top (right) side of the cable 


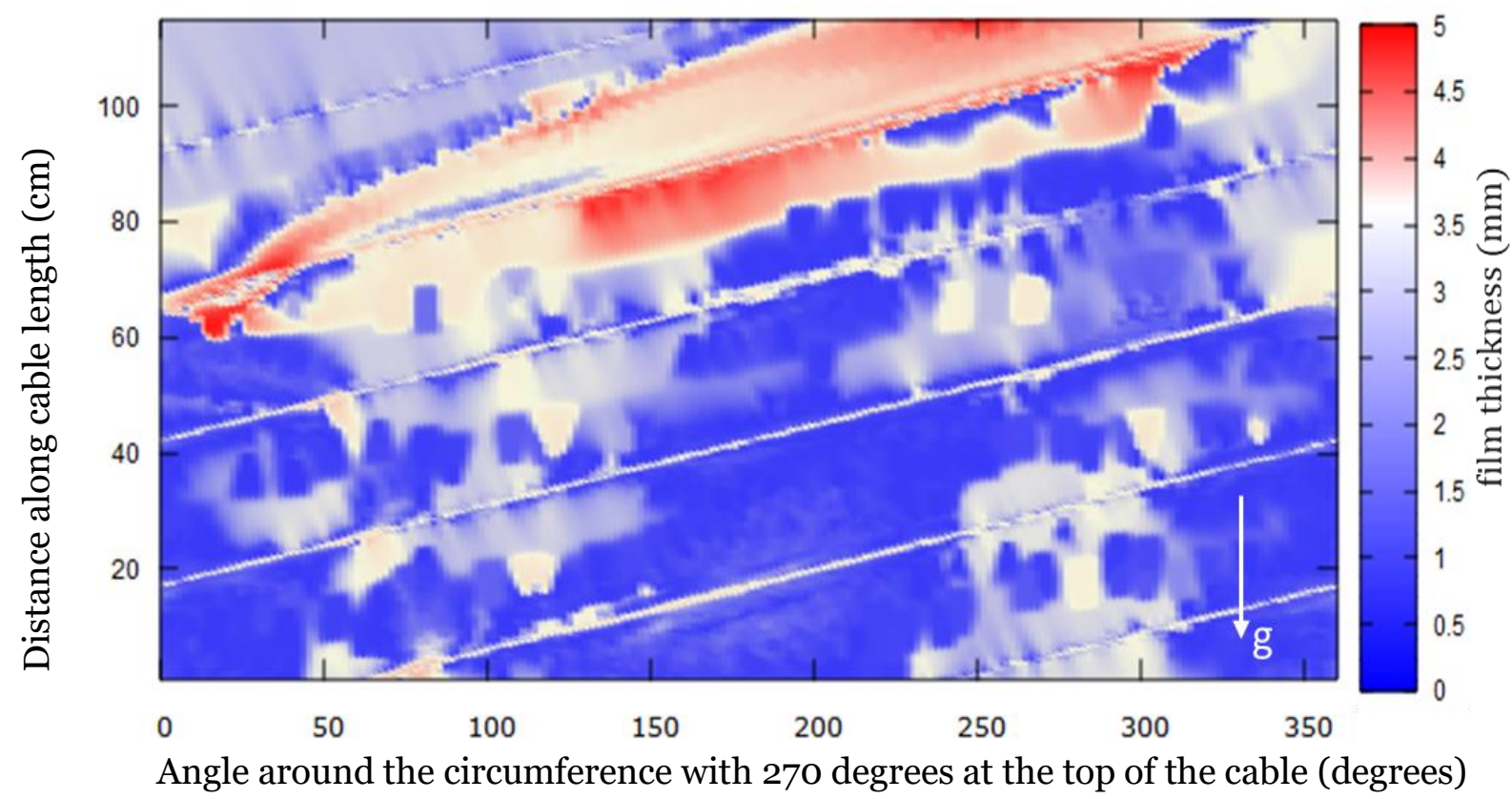

Figure 4-8 Rivulet and film thickness on cable with fillets. The vertical axis denotes the length along the axis of the cylinder while the bottom axis denotes the angle along the circumference of the cylinder with angle 270 degrees at the top of the cable.

Although RANS modeling is able to form a fluid film on the cable, the film thickness is higher than expected. Film thickness should be in the range of 0.1 to $0.5 \mathrm{~mm}$ as observed in multiple experiments and numerical modeling [9, 10]. This behaviour of RANS modeling is not unusual as it computes near wall turbulence from time-averaged quantities. In the thin film flows with low Reynolds number, near wall flow (viscosity-affected region) is dominated by small length scale turbulence with small time scales. The RANS based standard k-epsilon model uses wall functions for this viscosity-affected region, which is based on a constant shear layer assumption [15]. In thin film flow with low Reynolds number, the constant shear layer assumption ceases to be valid [16] and the model tends to overestimate the eddy viscosity near the wall. Such situations require large eddy simulation or near-wall models that are valid for the viscosity affected region and that are integrable all the way to the wall.

\subsection{Lagrangian Transport Model}

In this case, adding and removing water from the water film was tested. The computational domain size, initial and boundary conditions are the same as in the previous case (section 3.1.1), except a rain source is used instead of water inflow from the top edge of the cable.

The rain was represented with a fully coupled Lagrangian transport model. The injection surface was located on the top surface of the domain, and covered a section right above the cable. The injection rate was again equal to $60 \mathrm{~mm} / \mathrm{hr}(2.4 \mathrm{in} / \mathrm{hr})$. The rain droplets were of mixed size, ranging from $0.2 \mathrm{~mm}$ to $2 \mathrm{~mm}$. About 100 thousand droplets of water were released from the injection surface during the simulation. 
Figure 4-9 shows rain droplets falling on the cable at simulated time $t=\sim 2$ sec. Figure 4-10 shows the upper $60 \%$ of the cable length, where the water film covers the cable. The small falling droplets of water slide down the curved surface of the cable and accumulate on the bottom of the cable forming a rivulet. A distinct elongated red streak can be seen on the plot in Figure 4-10. The thickness of the bottom rivulet is increasing along the cable axis as more water is being added to it. Figure 4-11 shows an instantaneous velocity magnitude of the water film on cable surface.

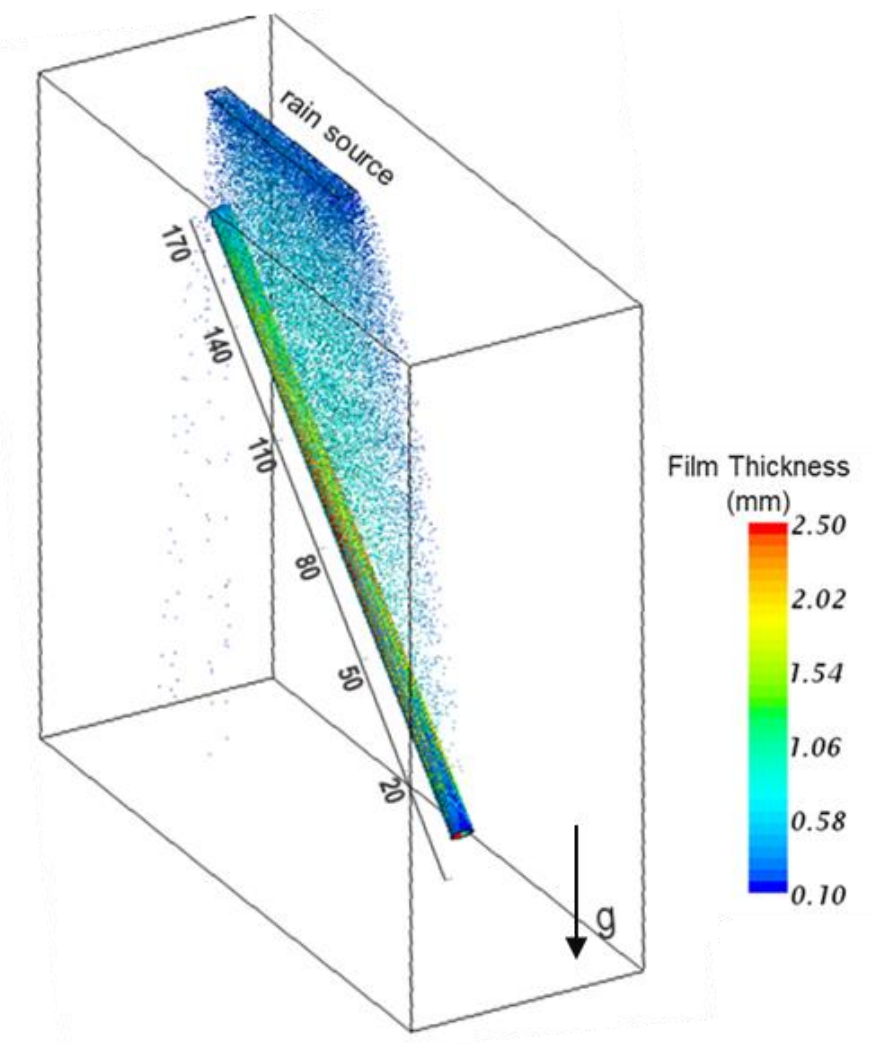

Figure 4-9 Injection of the Lagrangian particles into the domain, simulating rainfall 


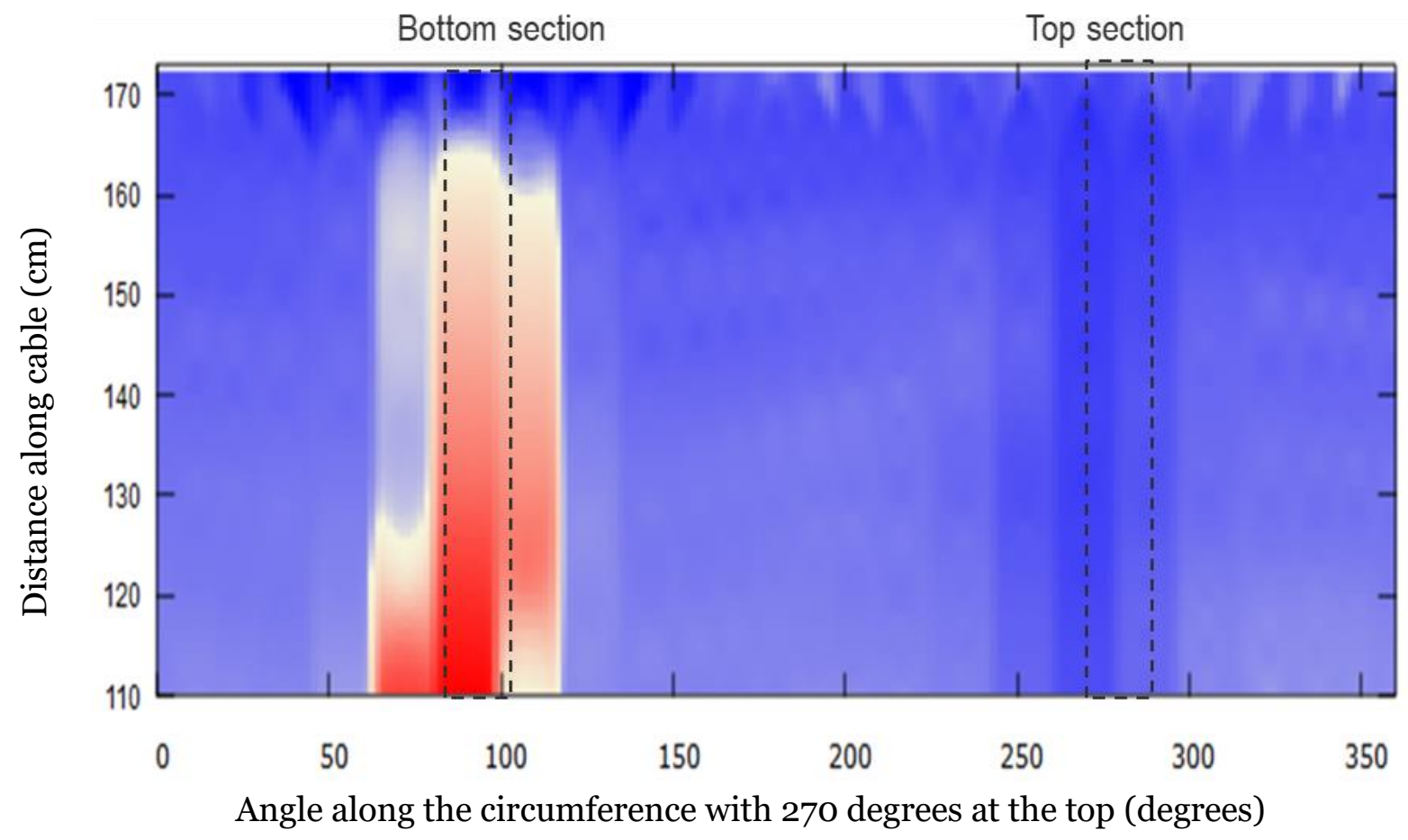

Figure 4-10 Fluid film thickness on the cable with fillets. The vertical axis denotes the length along the axis of the cylinder while the bottom axis denotes the angle along the circumference of the cylinder with angle 270 degrees at the top of the cable.

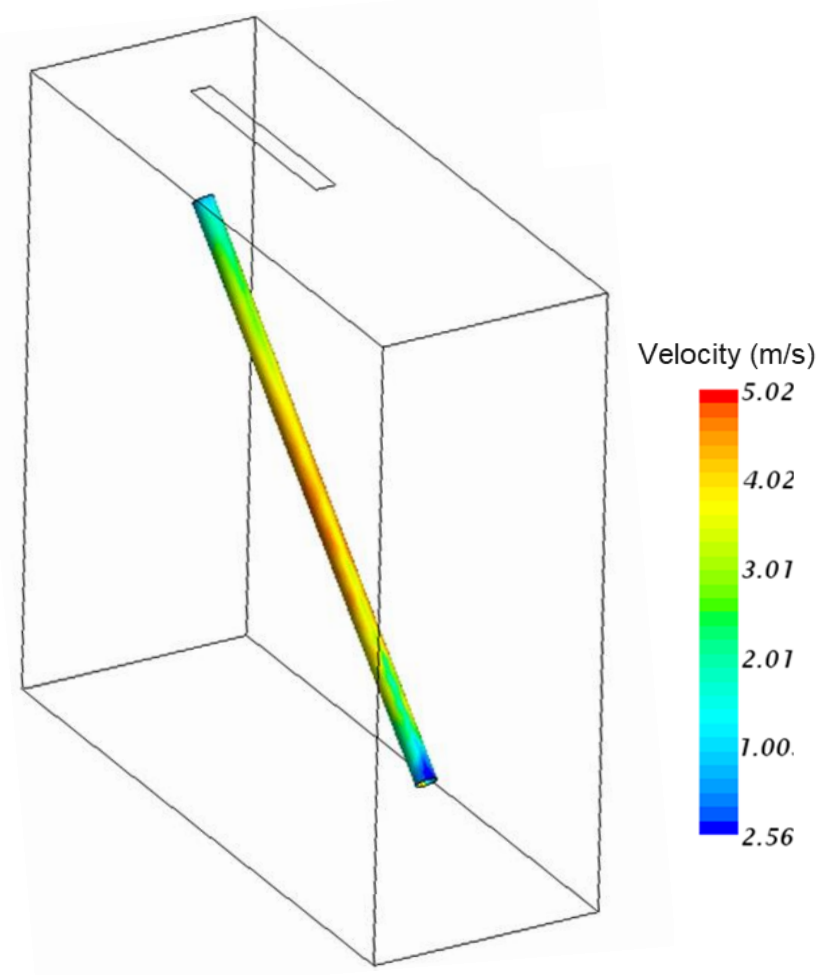

Figure 4-11 Velocity of water in the fluid film on the cable surface 
In Lagrangian transport modeling, each droplet is tracked independently via solution of an independent transport equation (ordinary differential equation) and this approach makes this type of simulation computationally very expensive for large numbers of droplets, of the order of hundreds of thousands. As the primary focus of this study was to analyze the mechanism of rivulet formation and flow on a cable surface and not to simulate the rain, other mechanisms that allow for the addition of water to the cable are explored in the following section. This test did show, however, that rain falling through air and collecting on a surface under the influence of gravity can be modeled if sufficient computational resources are available.

\subsection{Mass Source Term}

According to the findings presented in previous chapters, RANS based models are unable to predict the flow physics in the water film and in the wake of the cable sufficiently well. Therefore, Large Eddy Simulation was tested for simulating the rivulet formation and flow.

An extended domain, as compared to the models from the initial phase of the study, was used in the simulations described in this section. The overall domain length is $30 \mathrm{D}$, with $7 \mathrm{D}$ in front and 22D in the downstream, where $\mathrm{D}$ is the cable diameter. The sideways dimension is $24 \mathrm{D}$, and $7 \mathrm{D}$ distance is maintained in the vertical direction from the top and bottom faces of the cable. These dimensions were chosen to avoid boundary condition assumption effects at the bounding box boundaries on the near wake field solutions. The model represents a section of bridge cable with helical fillets with the same geometry as described in section 4.2. The cable was positioned at an angle to the horizontal surface and it was parallel to the inflow surface of the domain, as shown in Figure 4-12.

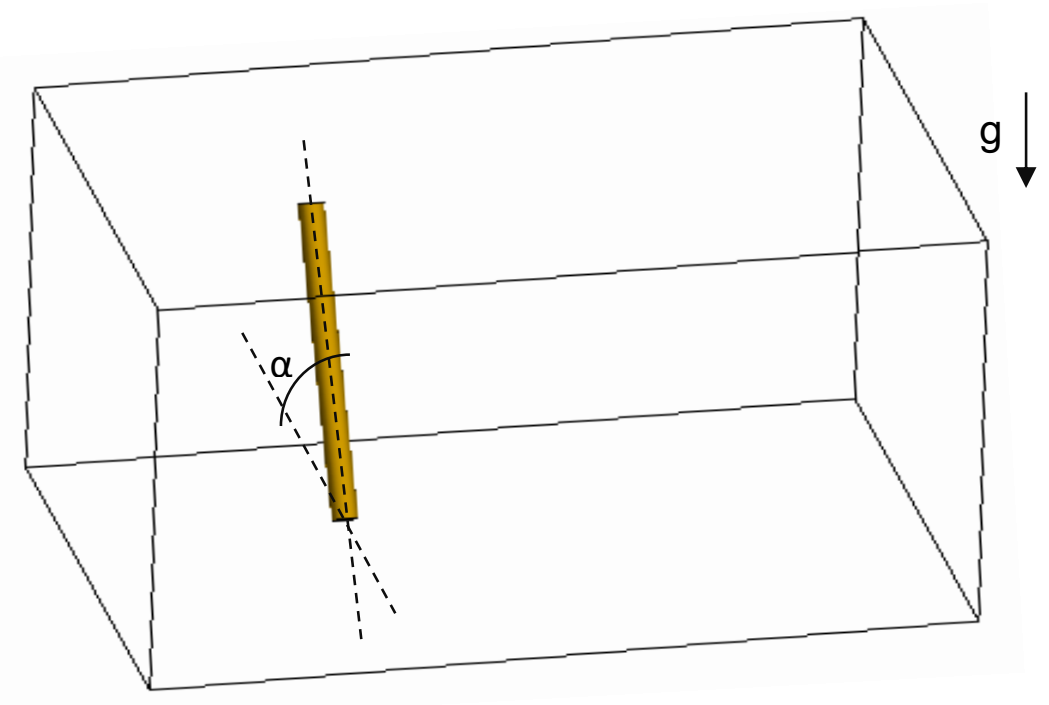

Figure 4-12 Computational domain used in this part of the study 
A trimmed mesh scheme was employed to generate a smooth and sufficiently fine grid in the domain. Twenty grid points were used in the first $10 \mathrm{~mm}$ near the cylindrical cable surface with hyperbolic stretching, and with the first grid point 40 microns away from the surface. This grid spacing resulted in $\mathrm{y}+=\sim 1$ in the critical areas on the cable surface. This condition is sufficient to compute the wall shear stress from the definition. The grid size close to the cable surface in the tangential and axial directions ranged from $3 \mathrm{~mm}$ to $4 \mathrm{~mm}$ and the size increased away from the surface. The total number of the computational cells used in these simulations was approximately 11 million. Snapshots of the mesh on the cable surface and fillets are shown in Figure 4-13.
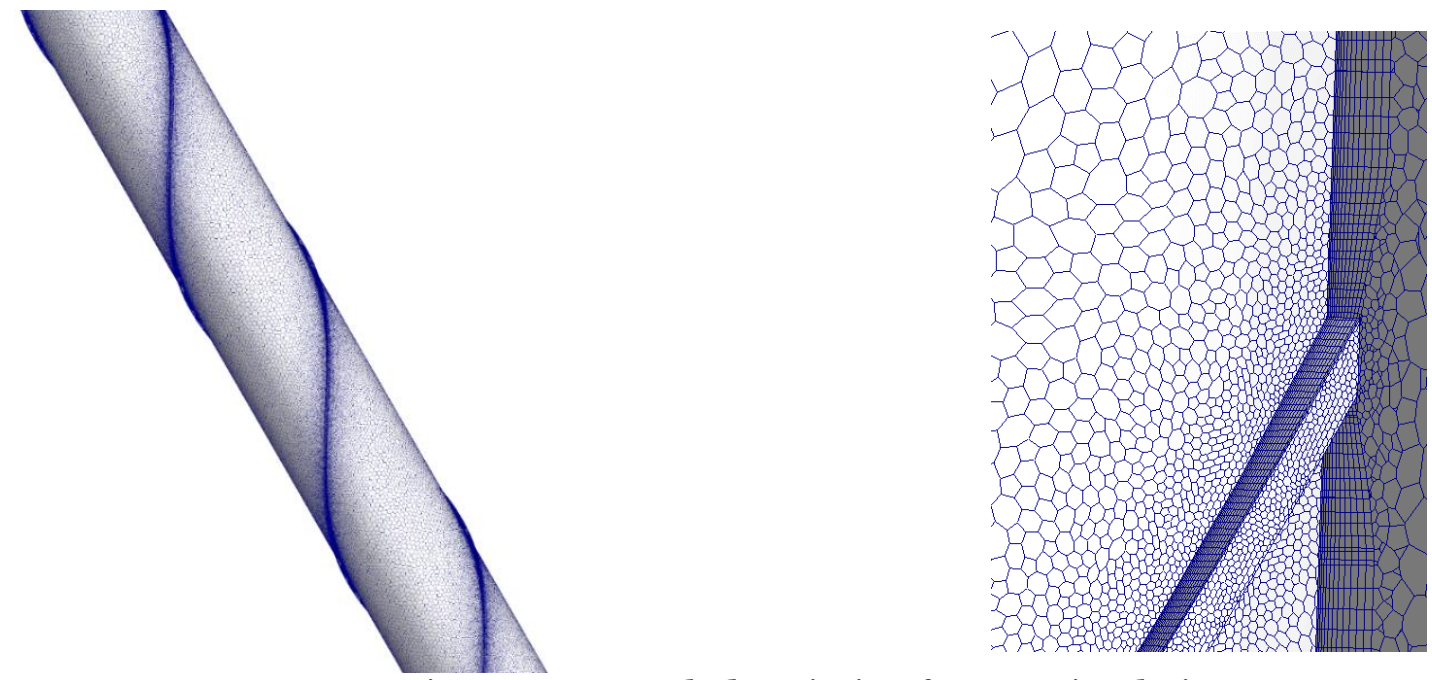

Figure 4-13: Mesh description for LES simulations

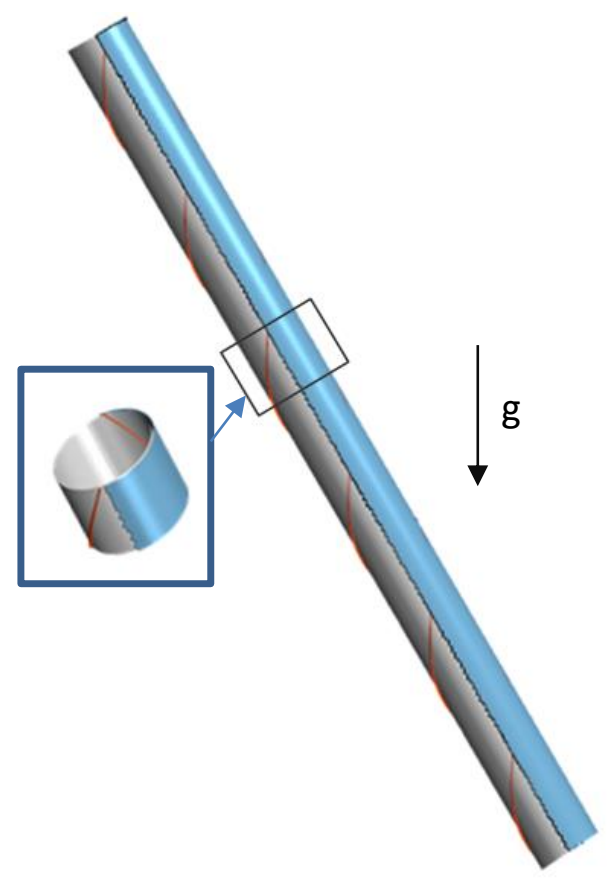

Figure 4-14 Location of the mass source term region for rain shown in blue 
A multiphase Eulerian model was used in the LES simulation. The air-water interface was modeled with the VOF model, as in the RANS simulations described in the previous sections. Unlike the RANS simulations, no inflow surface was used. Instead, a mass source term was employed on the upper section of the cable to mimic the effect of rain droplets accumulating on the cable surface. Figure 4-14 shows the mass source term region near the cable top surface (marked in blue).

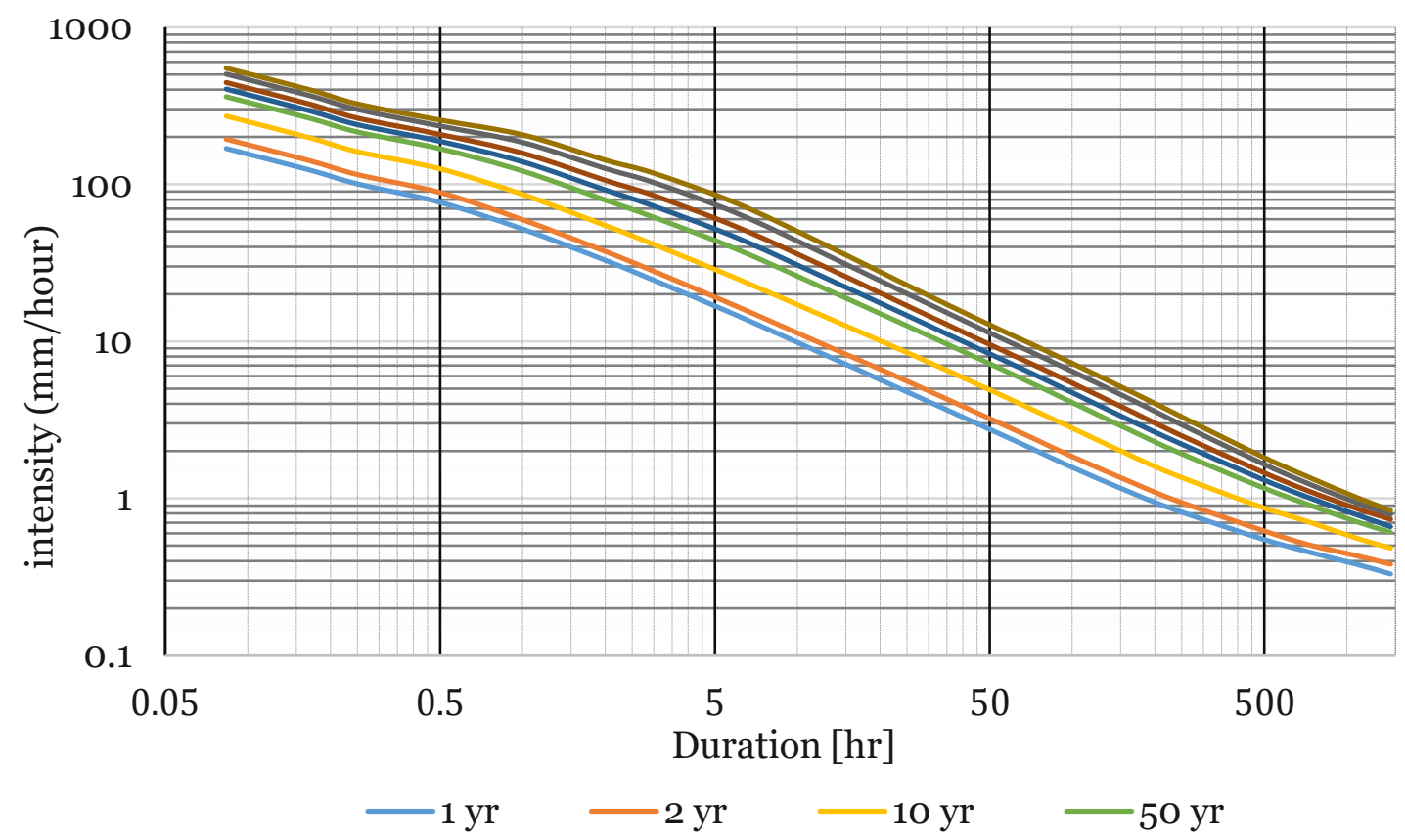

Figure 4-15 Rainfall intensity vs duration curves for various events in Louisiana. Source: http://hdsc.nws.noaa.gov/hdsc/pfds/

The simulations were performed for three rain rate events: $30 \mathrm{~mm} / \mathrm{hr}(1.2 \mathrm{in} / \mathrm{hr}), 60 \mathrm{~mm} / \mathrm{hr}(2.4$ $\mathrm{in} / \mathrm{hr}$ ), and $400 \mathrm{~mm} / \mathrm{h}(16 \mathrm{in} / \mathrm{hr})$ corresponding to 1-year, 2-year, and 200-year rainfall events. The $30 \mathrm{~mm} / \mathrm{hr}$ and $60 \mathrm{~mm} / \mathrm{hr}$ events are based on a 1-hour rain duration while the 400-year rain is based on a 6-minute rain duration. The data used is based on the rainfall intensity vs duration curves for Louisiana, as shown in Figure 4-15.

Although it is possible to resolve all the features of a liquid film, e.g., residual layers and capillary waves, using VOF and LES, the accuracy of the solution depends on the grid resolution. The grid used in this simulation was sufficiently refined to resolve most of the wall normal components of the flow and turbulence, but not refined enough in the tangential and axial direction to resolve the sub-grid scale features. The unresolved subgrid-scale features are modeled using an eddy viscosity based (wall adaptive local eddy viscosity (WALE)) model.

\subsection{1. $30 \mathrm{~mm} / \mathrm{hr}$ Rain Intensity}

In this simulation, the film formation on a stay-cable in low-intensity rain $(30 \mathrm{~mm} / \mathrm{hr})$ was studied. Figure 4-16 and Figure 4-17 show the water film with no crosswind at time $t=\sim 1 \mathrm{~s}$ under Modeling of Water Film Formation on a Stay-Cable 
the influence of gravity and rain entering via a mass source term. For low rain rates, the inclination of the cable causes small droplets of water to slide on the curved surface and form a thin film. The water accumulates along the fillets and falls off after overtopping them. Figure 4-16 shows the entire surface of the cable. The cross lines in this figure represent the fillets on the cable surface. Near the lower section of the cable, between 50 and 90 degrees, relatively thicker water streaks form. The rivulets have a thickness between $0.5 \mathrm{~mm}$ and $1 \mathrm{~mm}$, which is consistent with the experimental measurements of $\mathrm{Li}$ et al [17]. These streaks form as the surface tension forces cannot overcome the gravitational forces. Also, several red spots on the top part of the cable represent the overtopping and separation of the film from the outer edge of the fillets, which then drops away through the air. In all other locations on the cable, a thin film is formed on the surface with a thickness less than $0.2 \mathrm{~mm}$ due to the rain mass source condition.

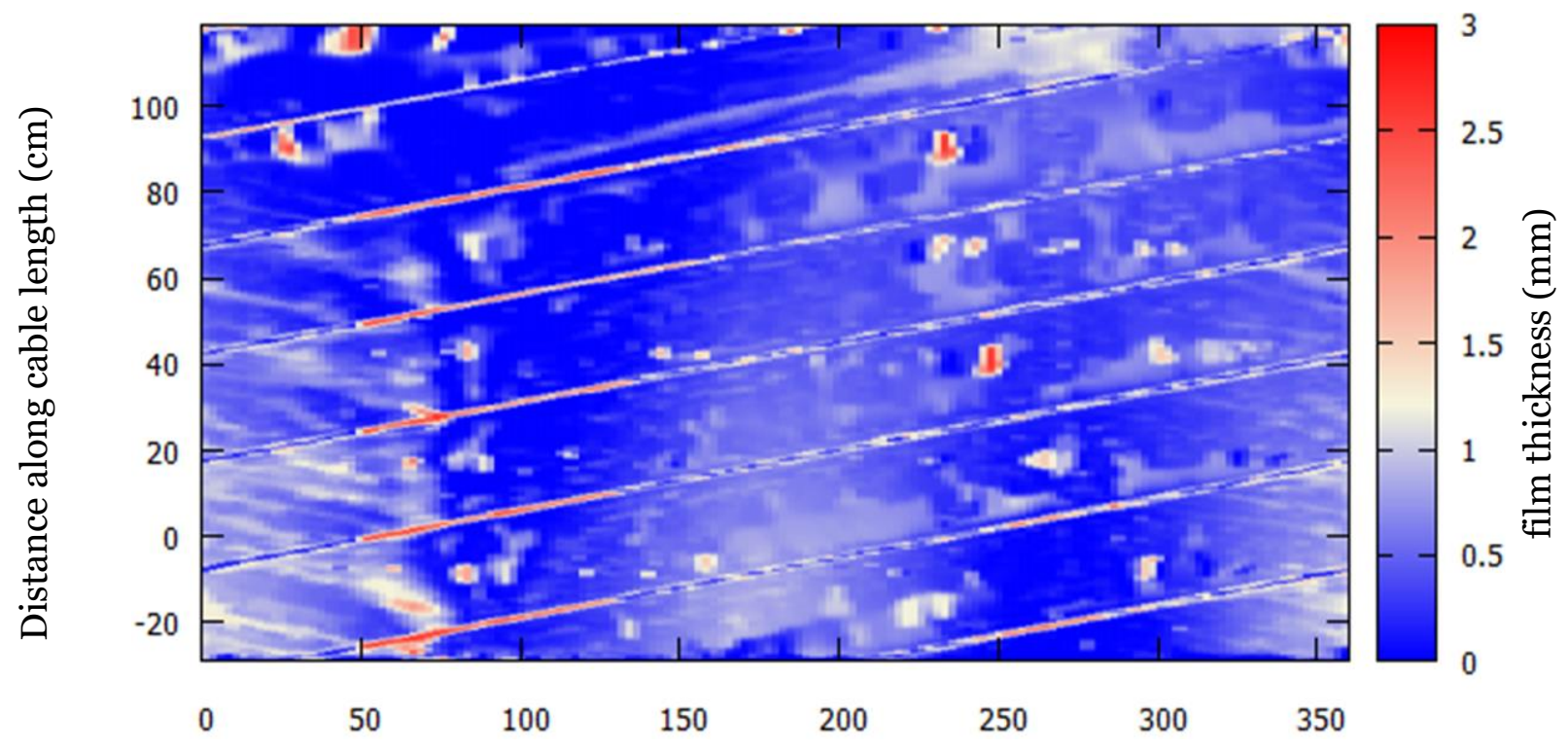

Angle around the circumference with 270 degrees at the top of the cable (degrees)

Figure 4-16 Fluid film thickness on the cable for $30 \mathrm{~mm} / \mathrm{hr}(1.2 \mathrm{in} / \mathrm{hr})$ rain. The vertical axis denotes the length along the axis of the cylinder while the bottom axis denotes the angle along the circumference of the cylinder with angle 270 degrees at the top of the cable 


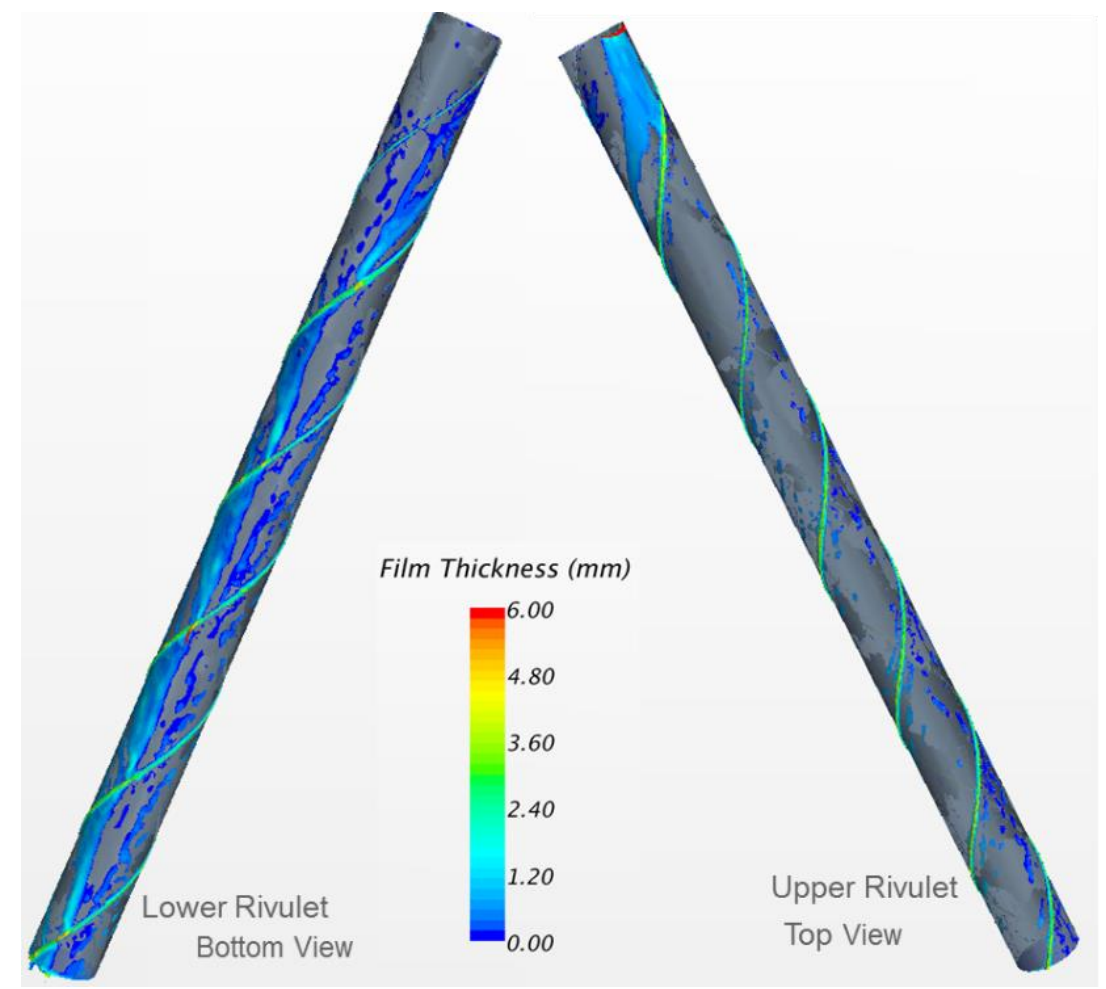

Figure 4-17 Rivulets on the bottom (left) and top (right) side of the cable

\subsection{2. $60 \mathrm{~mm} / \mathrm{hr}$ Rain Intensity}

For a two year rain event with rain intensity of $60 \mathrm{~mm} / \mathrm{hr}(2.4 \mathrm{in} / \mathrm{hr})$ simulation, the water film on stay-cable is shown in Figure 4-18. The mechanism of the formation of lower rivulets is similar to that observed for the $30 \mathrm{~mm} / \mathrm{hr}(1.2 \mathrm{in} / \mathrm{hr})$ rain case. However, the effect of the high-intensity rain can be easily viewed in terms of coherency of the streak and rivulet structures. The figure shows a slightly thicker and more continuous streak (lower rivulet) of water in the region between 60 and 80 degrees, as compared to the $30 \mathrm{~mm} / \mathrm{hr}$ rain case. This streak can also be seen in Figure 4-19. The position of the lower rivulets is consistent with measurements of Chen et al [6]. On the upper side of the cable, except for a few elongated streaks, no continuous film is visible, suggesting the absence of the upper rivulets. Apart from these, raindrops sliding along the sides of the cable can also be seen in the region near the o degrees ( \pm 30 degrees $)$ and 180 degrees $( \pm 30$ degrees) sections. This sliding film has a thickness of less than $0.3 \mathrm{~mm}$. 


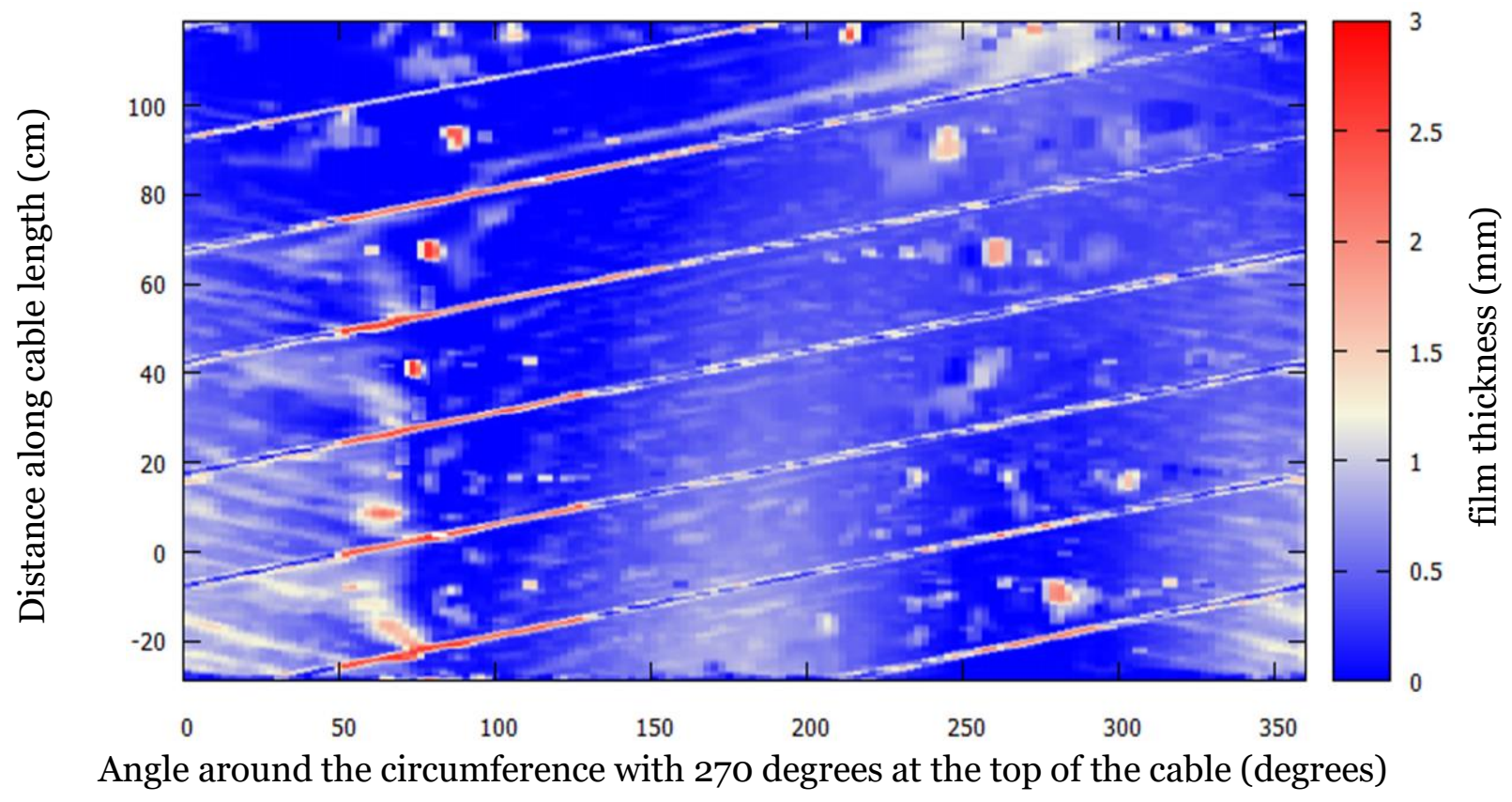

Figure 4-18 Fluid film thickness on the cable for $60 \mathrm{~mm} / \mathrm{hr}(2.4 \mathrm{in} / \mathrm{hr})$ rain intensity. The vertical axis denotes the length along the axis of the cylinder while the bottom axis denotes the angle along the circumference of the cylinder with angle 270 degrees at the top of the cable.

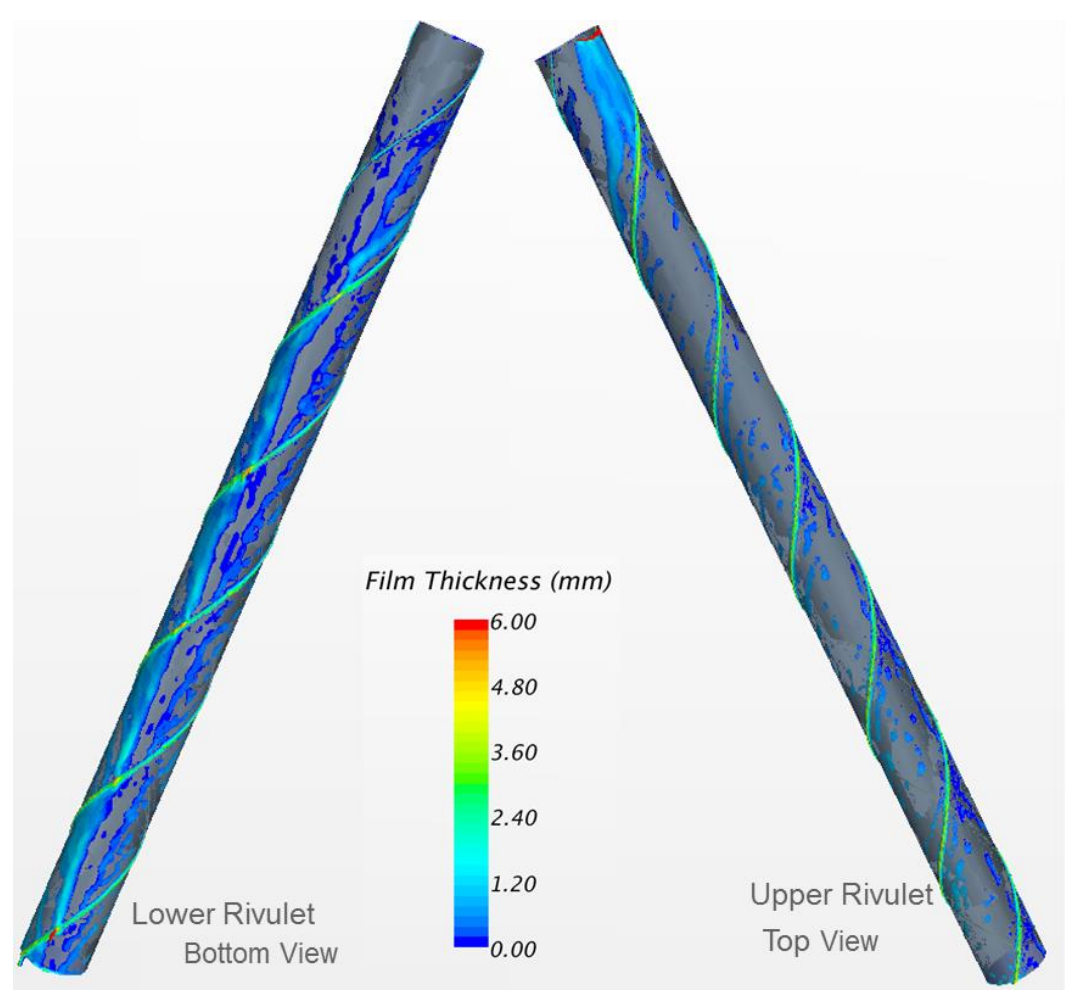

Figure 4-19: Rivulet formation on the bottom (left) and top (right) side of the cable. 


\subsubsection{0 mm/hr Rain Intensity}

The 200 year $(400 \mathrm{~mm} / \mathrm{hr})$ rain event represents a strong downpour scenario. For this case, a water film with distinctive streaks is shown in Figure 4-20. This extreme rain event produces a much thicker water film, which increased by 5 to 6 times as compared to the $30 \mathrm{~mm} / \mathrm{hr}$ and 60 $\mathrm{mm} / \mathrm{hr}$ rain events. This extreme event did not generate any upper rivulets on the cable, see Figure 4-21, which supports the observations of other researchers $[3,18,19]$ (that the upper rivulets can only appear in the presence of a crosswind. The thickness of the lower rivulet is about $2.4 \mathrm{~mm}$ in this case and is limited by the height of the fillets. Any water beyond the $2.4 \mathrm{~mm}$ thickness in the film is shed off at the edge of the fillet under the influence of gravity.

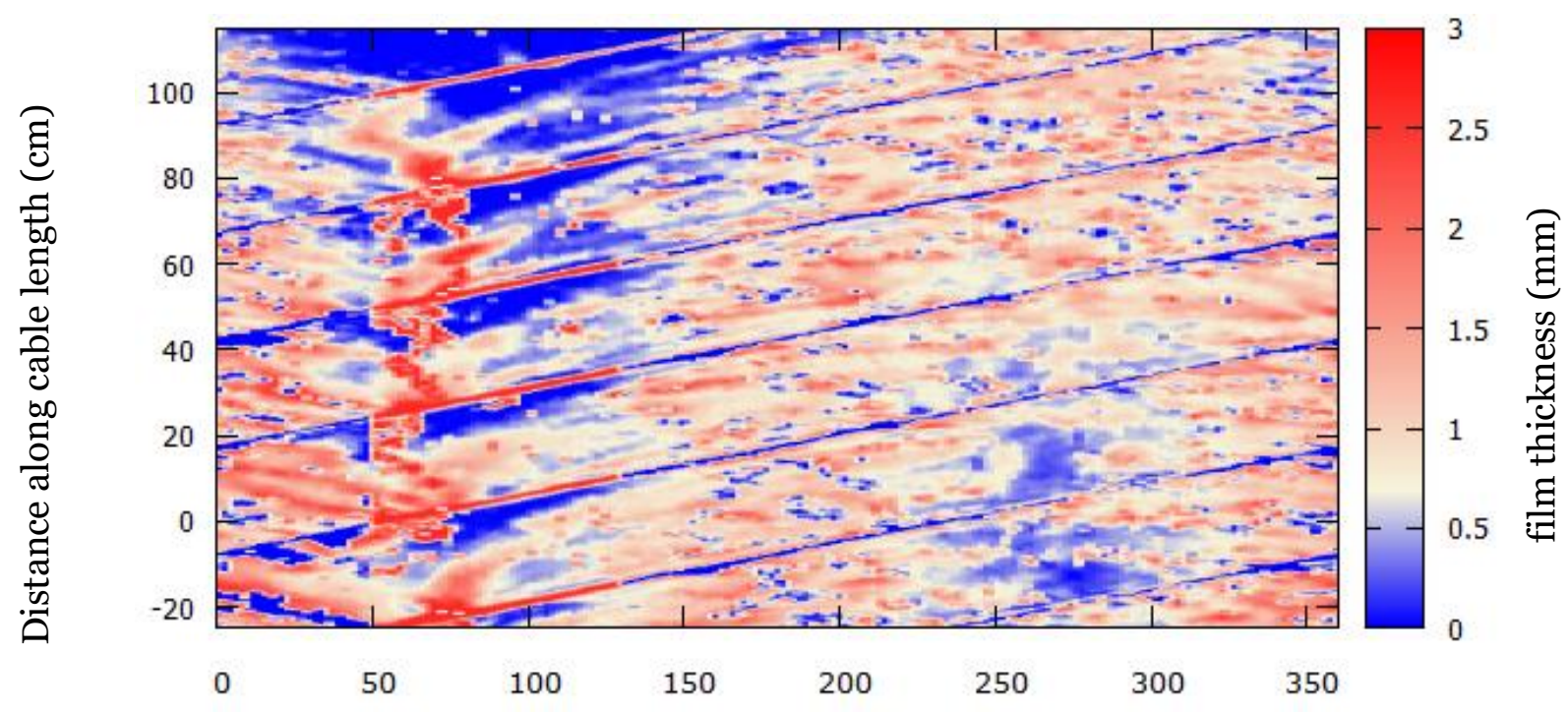

Angle around the circumference with 270 degrees at the top of the cable (degrees)

Figure 4-20 Fluid film thickness on the cable for $400 \mathrm{~mm} / \mathrm{hr}$ rain. The vertical axis denotes the length along the axis of the cylinder while the bottom axis denotes the angle along the circumference of the cylinder with angle 270 degrees at the top of the cable 


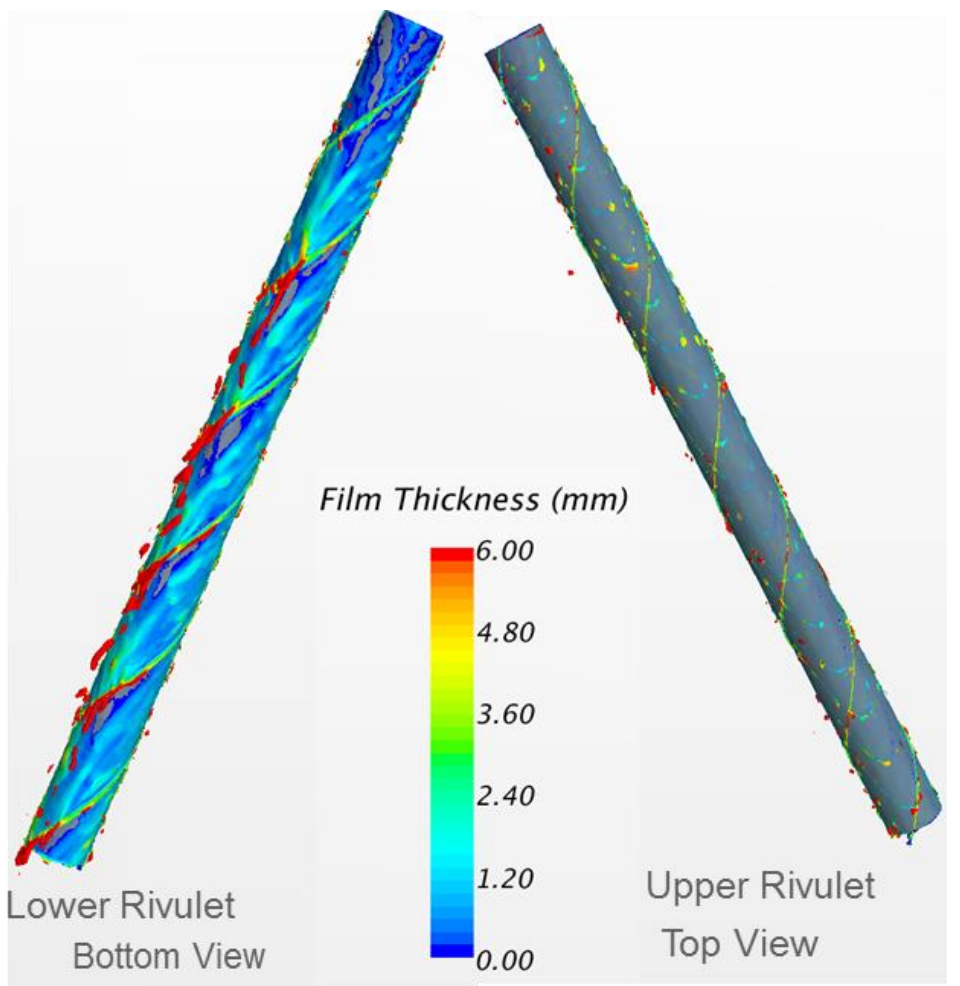

Figure 4-21: Rivulet formation on the bottom (left) and the top (right) end of the cable

\section{Influence of Crosswind Flow on Rivulet Formation and Flow}

In this section, we examine modeling of rivulet formation in crosswind flows and analyze the change in aerodynamic behaviour of the cable with and without fillets. First, the aerodynamic behaviour of the cable with and without fillets for dry conditions (with no inflow of the fluid) was analyzed. Then, the flow over a cable in wet conditions in several crosswind flows was studied. For the cases in wet conditions, water was introduced into the domain with the use of a mass source on the cable surface. The size of the domain was the same as described in section 4.4 and presented in Figure 4-12. The boundary surface on the left side of the domain was assigned an inlet velocity type of a boundary condition, through which air enters the domain with specified velocity to simulate wind. Pressure boundary was assigned to the right side of the domain, and the other surfaces were assigned a symmetry boundary condition.

\subsection{Instantaneous and Mean Flow Field in Dry Conditions}

A steady crosswind flow with speed $6.2 \mathrm{~m} / \mathrm{s}$ and angle of attack zero degrees was analyzed. The mesh used in this study was the same as shown in Figure 4-13. Figure 5-1 presents an instantaneous vorticity magnitude field around the cable in dry conditions. The figure illustrates the effect of the fillets on the von Karman vortex street. They trigger an earlier, than in the case without fillets, separation of flow which results in a larger wake size. This early separation can also be seen in Figure 5-2. Figure 5-2 (a) shows the flow on a plain cylinder with no fillets. The 
structures form at a point of separation that is near 90 degrees from the downwind direction. This separation zone is also visible in the shear stress plot in Figure 5-3, as separation occurs at the point of zero shear stress. For the case of a cable with fillets, the flow separation is triggered by the presence of fillets and separation zone is located along them. Figure 5-3 shows the zero shear stress (dotted blue) line along the fillet on the cylinder surface. Multiple separations and reattachments of inner shear layer occur at the location where fillets are at a smaller angle from the downwind flow.
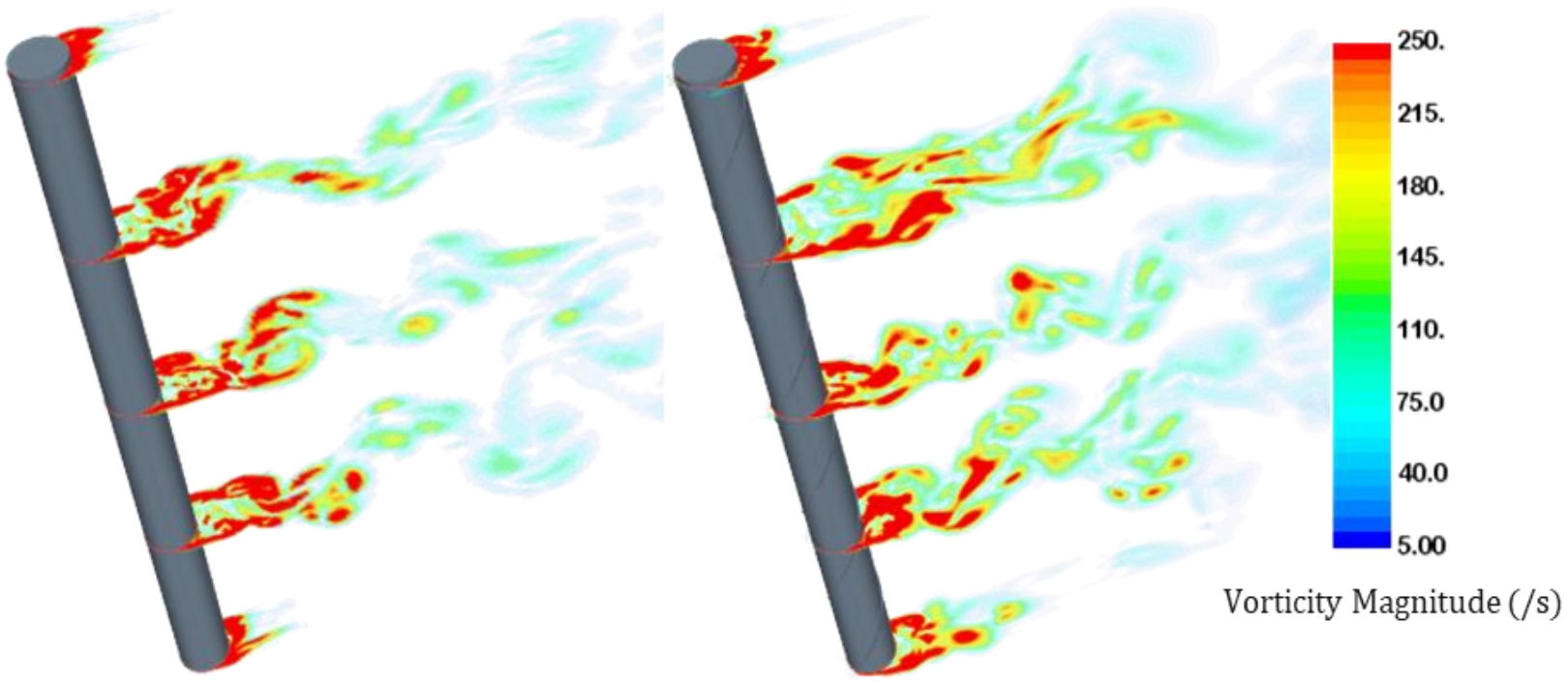

Figure 5-1 Vorticity magnitude for crosswind flow over cable in dry conditions, (a) plain cylinder and (b) cylinder with filllet

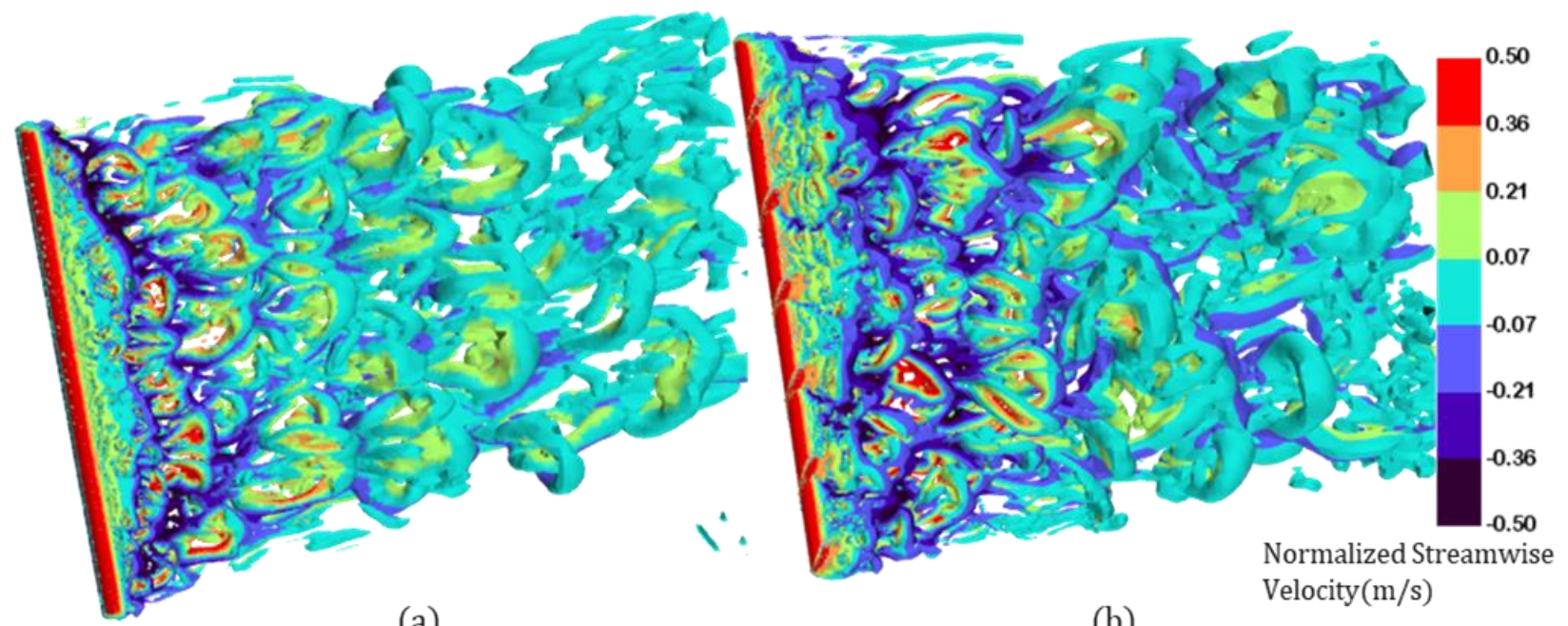

(a)

(b)

Figure 5-2 Coherent structures based on q-criteria with no fillets (left) and with fillets (right) 


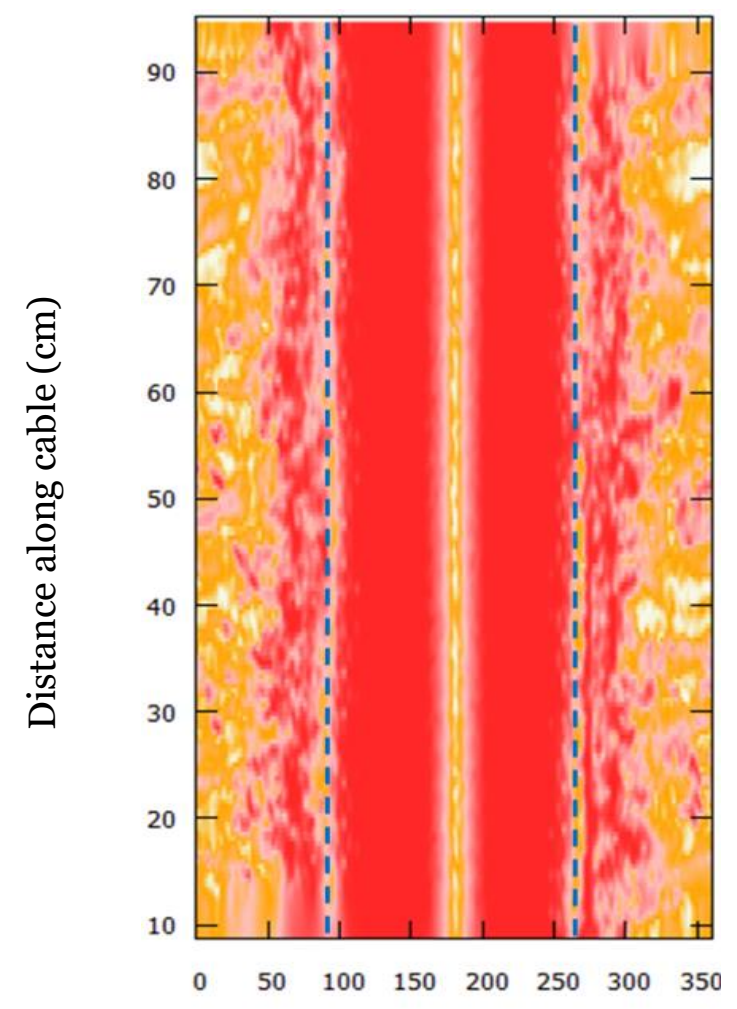

(a) without fillets

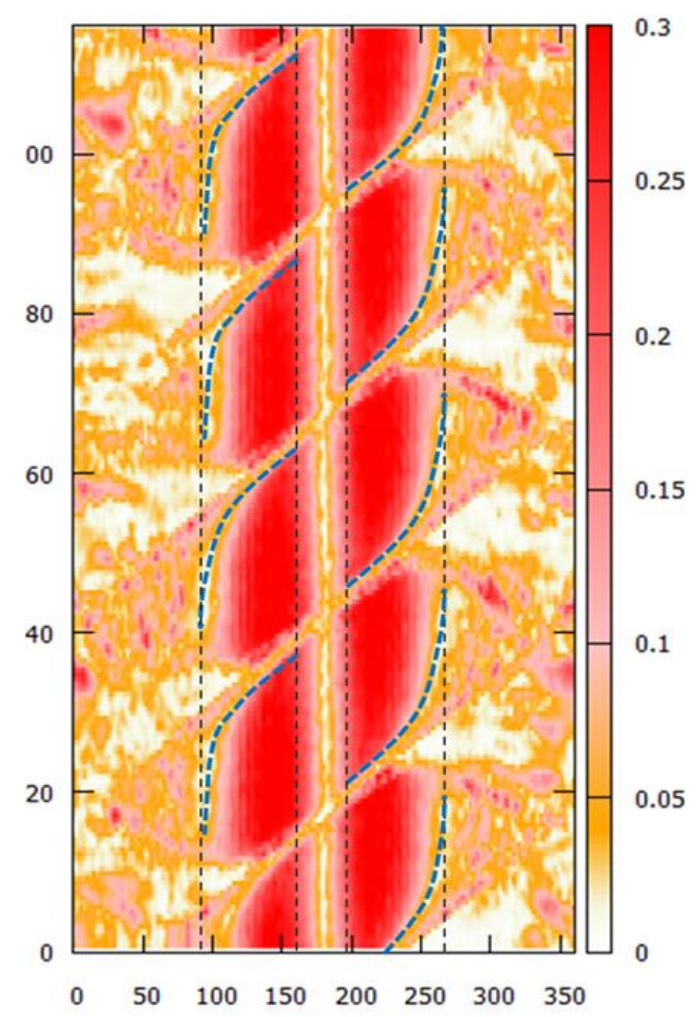

(b) with fillets

Figure 5-3 Shear stress distribution at wind velocity $6.2 \mathrm{~m} / \mathrm{s}$ on the surface of a (a) plain cylinder, and (b) cylinder with fillets. The vertical axis is distance along the axis of the cylinder while the bottom axis is the angle along the circumference of the cylinder with angle o degrees on the leeward side of the cable.

(a)

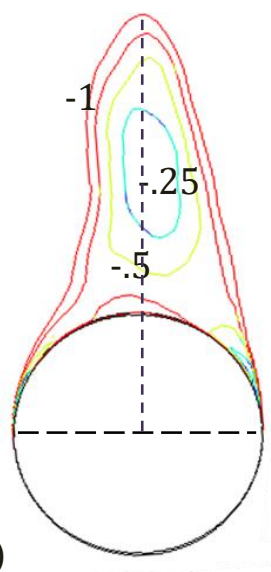

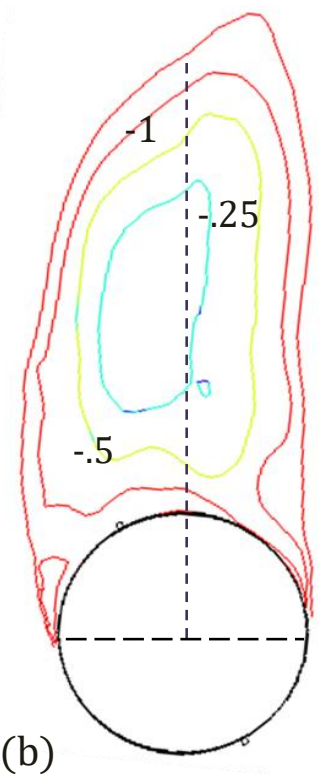

(b) (c)

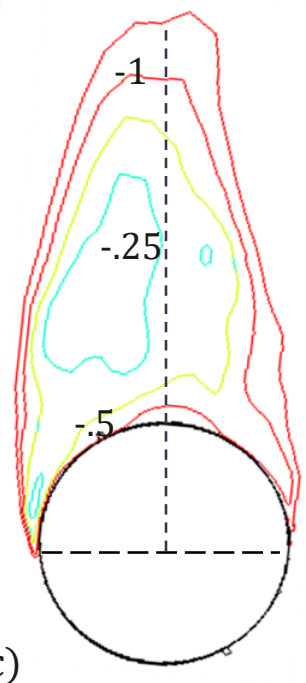

Figure 5-4 Contours of normalized downstream velocity (non-positive on a cross section plane for (a) plain cylinder, (b) cylinder with fillet (c) wet cylinder with fillet 


\subsection{Drag Coefficient}

Figure 5-4 shows isolines of the mean velocity in the streamwise direction representing the scale of the near wake recirculation region. The extent of this region is known as a vortex formation length and is defined as a distance from the centroid of the cable to a point where the centerline crosses the downstream end of the near wake zone. The formation length can be used to compare the relative drag force coefficient for different cable and flow configurations [20]. In Figure 5-5, the formation length is shown for three cases. For the plain cylinder (short cylinder $\mathrm{L} / \mathrm{D}=\sim 17$ ) the obtained drag coefficient is $\sim 1.04$, which is in the range of experimental values of Nakayama and Boucher [21] and Kleissl and Georgakis [5]. The introduction of a fillet increases the drag coefficient to about 1.4. Although it is hard to find any experimental data or other evidence to support the obtained value for the particular fillet and flow velocity, the addition of a fillet should increase the drag coefficient of the cable [14]. This increase is also evident from the increase in the vortex formation length. In the wet cable case, the water film on the surface acts as a lubricant and therefore delays the boundary layer transition and results in a drag reduction. This lubricating behaviour of fluid is similar to laminar flow control [22] and only valid for slow moving fluid, as fast moving fluid can destabilize the thin film layer. In this case, the drag coefficient is equal to approximately 1.34 (Figure 5-5), and the vortex formation length is slightly smaller compared to the case of a dry cable with a fillet. Drag reduction due to the water film on the cable is also observed by Matteoni and Georgakis [23].

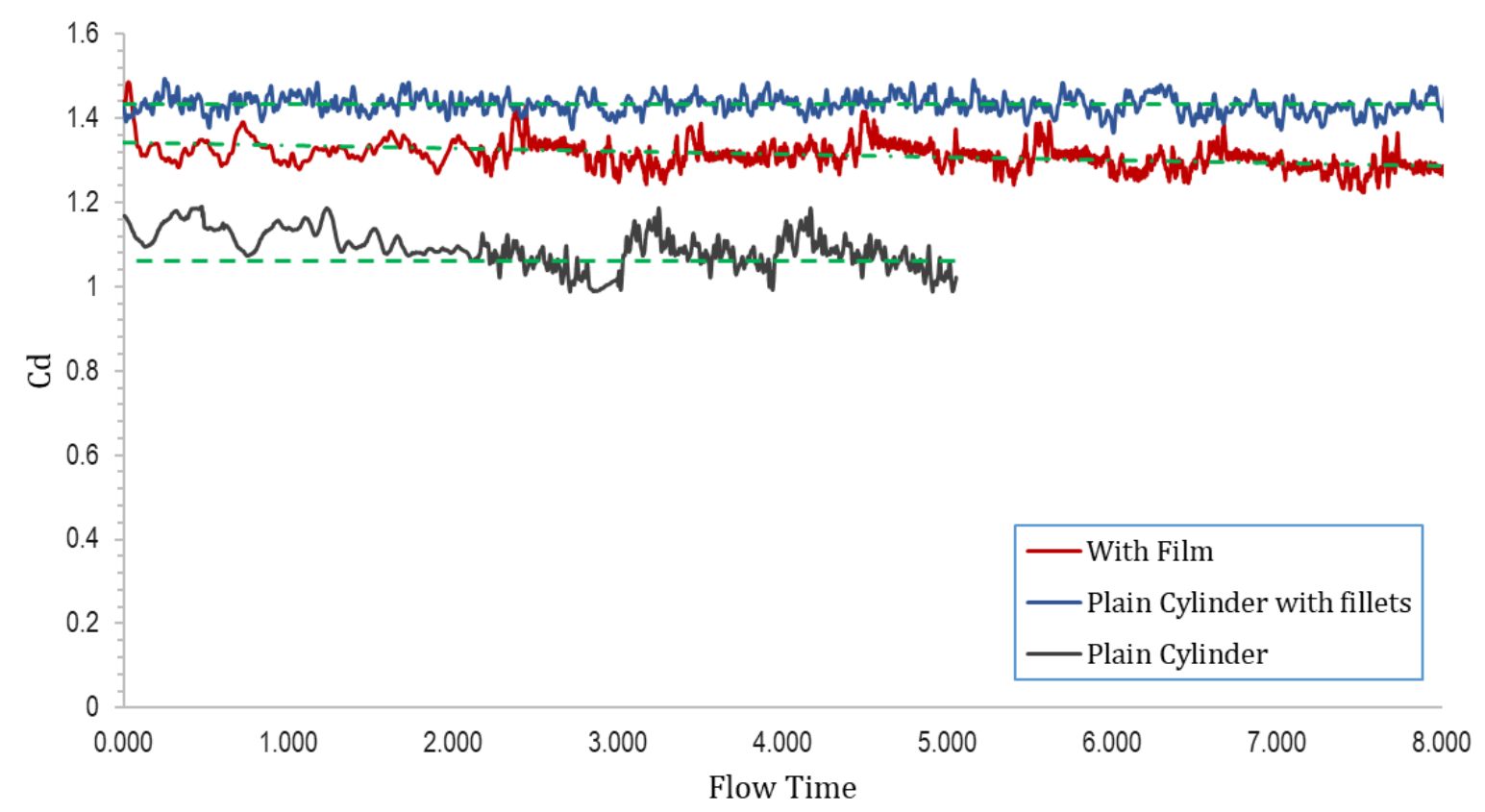

Figure 5-5 Time history of the drag coefficient, Cd, for cylinders: without fillets (black), with fillets (blue), and with fillets and water film (red) 


\subsection{Film Thickness and Upper Rivulet Formation}

In wet conditions with crosswinds, upper and lower water rivulets form on the cable surface when the wind velocity reaches value sufficient to overcome gravity in combination with the surface tension force. When the wind speed is very high, the rivulet formation gets disrupted as liquid droplets are blown off the surface due to the wind inertia.

The rivulet formation process is time-dependent. The rivulet configuration can be influenced by the incoming flow from the rivulets formed farther up on the cable. Figure 5-6 to Figure 5-10 show the water film surrounding the cable at a time instance of 2 seconds for wind speeds: $5 \mathrm{~m} / \mathrm{s}, 6.2$ $\mathrm{m} / \mathrm{s}, 7.2 \mathrm{~m} / \mathrm{s}, 7.7 \mathrm{~m} / \mathrm{s}$ and $8.7 \mathrm{~m} / \mathrm{s}$. In each of the figures, the water film thickness distribution on the cable surface is shown on the left, and surface film velocity on the top and bottom face of the cable is shown on the right.

Figure 5-6 shows the presence of upper rivulet on the cable for a wind velocity of $5 \mathrm{~m} / \mathrm{s}$. It forms due to the wind action on the fluid and follows a slightly sinusoidal trajectory along the cable length. There are two types of structures present in the upper rivulet: a thick streak called the 'main rivulet' and a less coherent secondary structure called a 'sub-rivulet'. The less coherent subrivulet looks like a mini tube flow and appears due to the air pushing the water film and therefore causing instability at the air-water interface also known as Rayleigh-Taylor instability. The maximum thickness of the lower rivulets is about $2.5 \mathrm{~mm}$ while the maximum upper rivulet thickness is less than $1.75 \mathrm{~mm}$. In the figure on the right, the surface velocity of the rivulet is shown.

For a crosswind velocity of $6.2 \mathrm{~m} / \mathrm{s}$, see Figure 5-7, the upper rivulet thickness increased, and the rivulet became more coherent. Also, the thickness of the lower rivulets decreased with an increase in wind speed and almost disappeared at a wind speed of $8.7 \mathrm{~m} / \mathrm{s}$ (see Figure 5-8, Figure 5-9, and Figure 5-10). The thickness of the upper rivulet, when the crosswind speed equals $8.7 \mathrm{~m} / \mathrm{s}$, is more than $3 \mathrm{~mm}$. This increase in thickness can be attributed to the fact that the rivulets separated from the cylinder surface near the fillets due to the strong wind. The upper rivulets for crosswind velocities $7.2 \mathrm{~m} / \mathrm{s}$ and $7.7 \mathrm{~m} / \mathrm{s}$ have a similar thickness, which is consistent with experimental measurements $[7,9]$. 

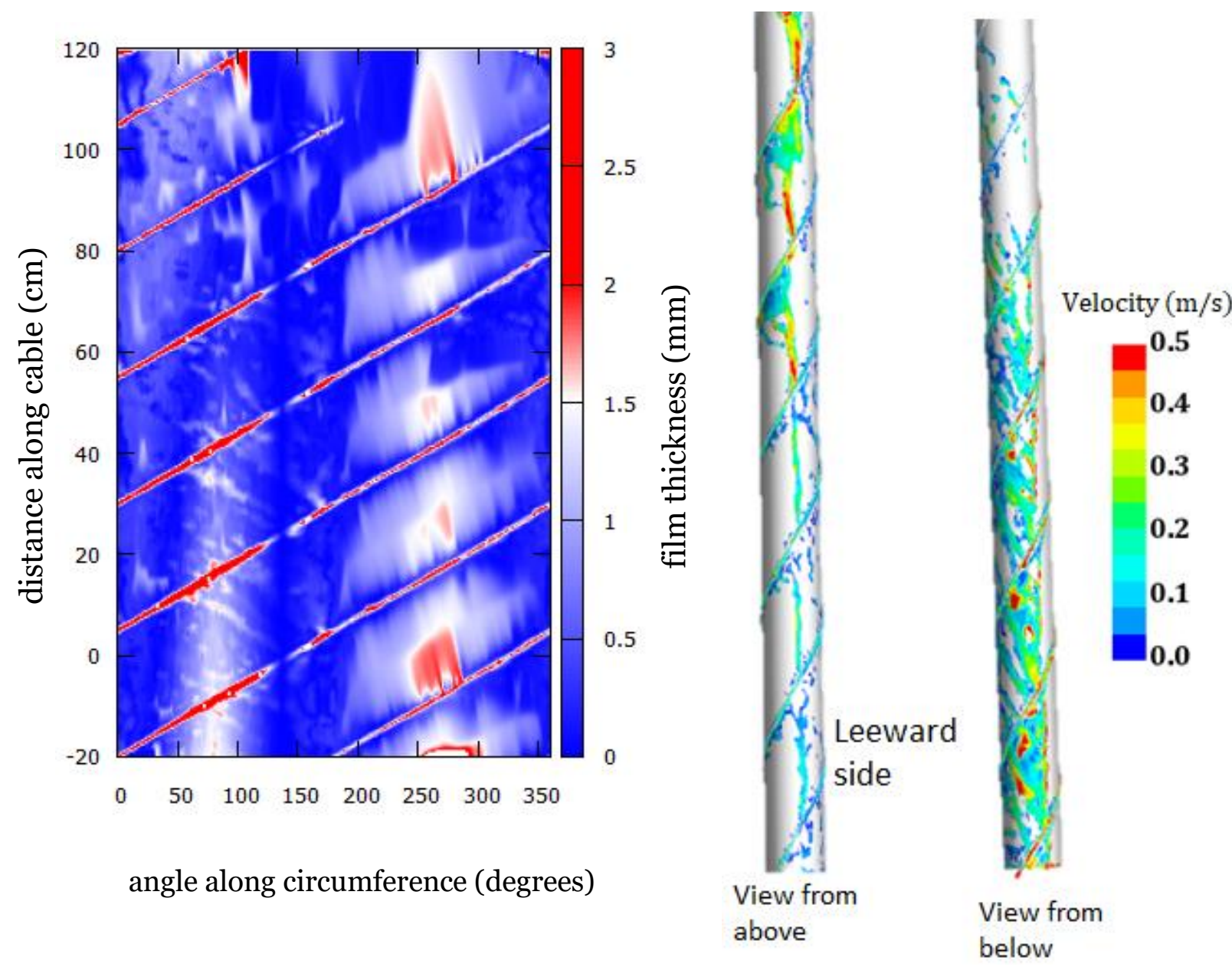

Figure 5-6 Wind velocity $5.0 \mathrm{~m} / \mathrm{s}$ and $60 \mathrm{~mm} / \mathrm{hr}$ (2.4 in/hr) rain: fluid film thickness on the cable surface (left), and film velocity (right). The vertical axis is the length along the axis of the cylinder while the bottom axis is the angle along the circumference of the cylinder with angle 270 deg at the top of the cable. 

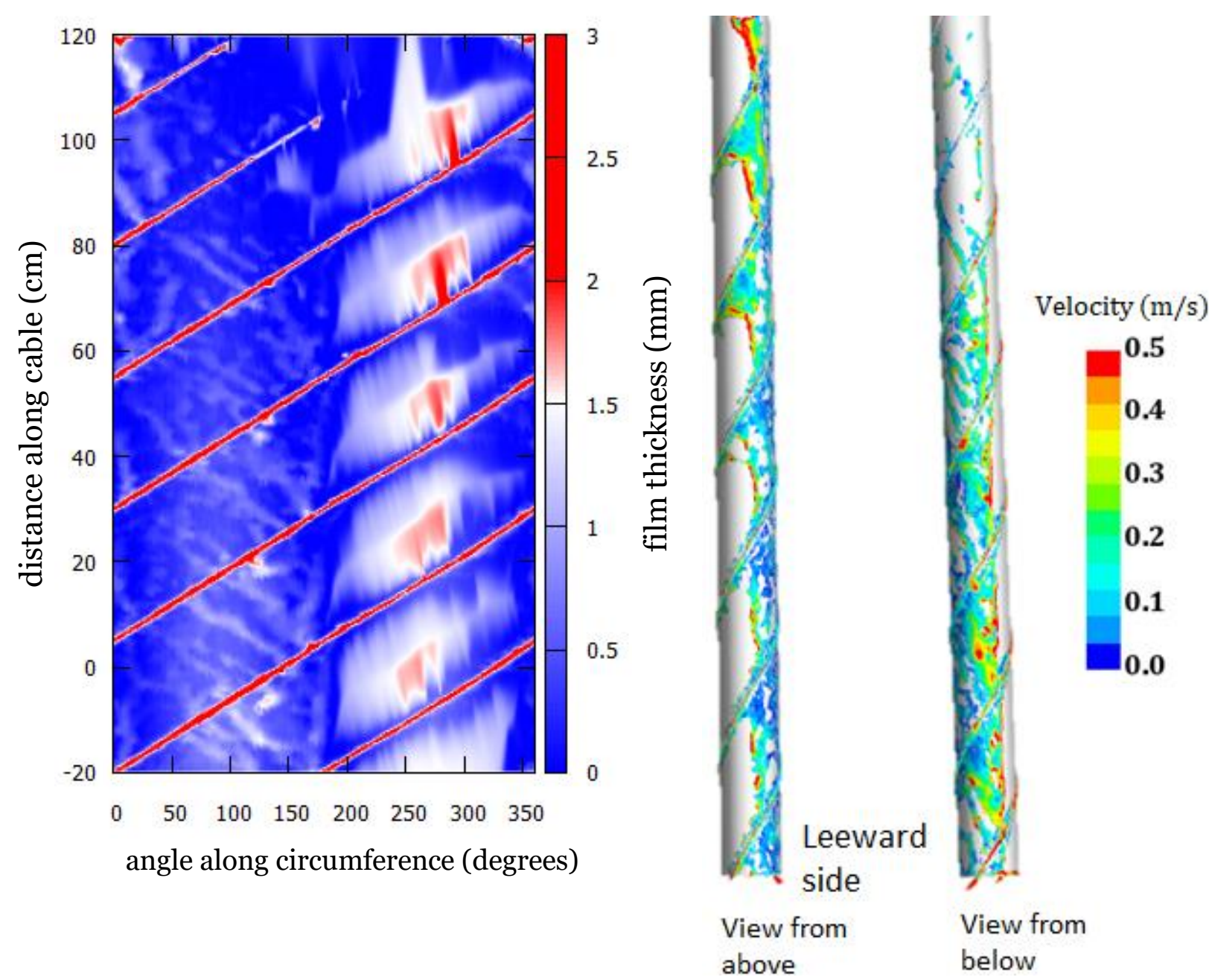

Figure 5-7 Wind velocity $6.2 \mathrm{~m} / \mathrm{s}$ and $60 \mathrm{~mm} / \mathrm{hr}(2.4 \mathrm{in} / \mathrm{hr})$ rain: fluid film thickness on the cable surface (left), and film velocity (right). The vertical axis is the length along the axis of the cylinder while the bottom axis is the angle along the circumference of the cylinder with angle 270 deg at the top of the cable. 


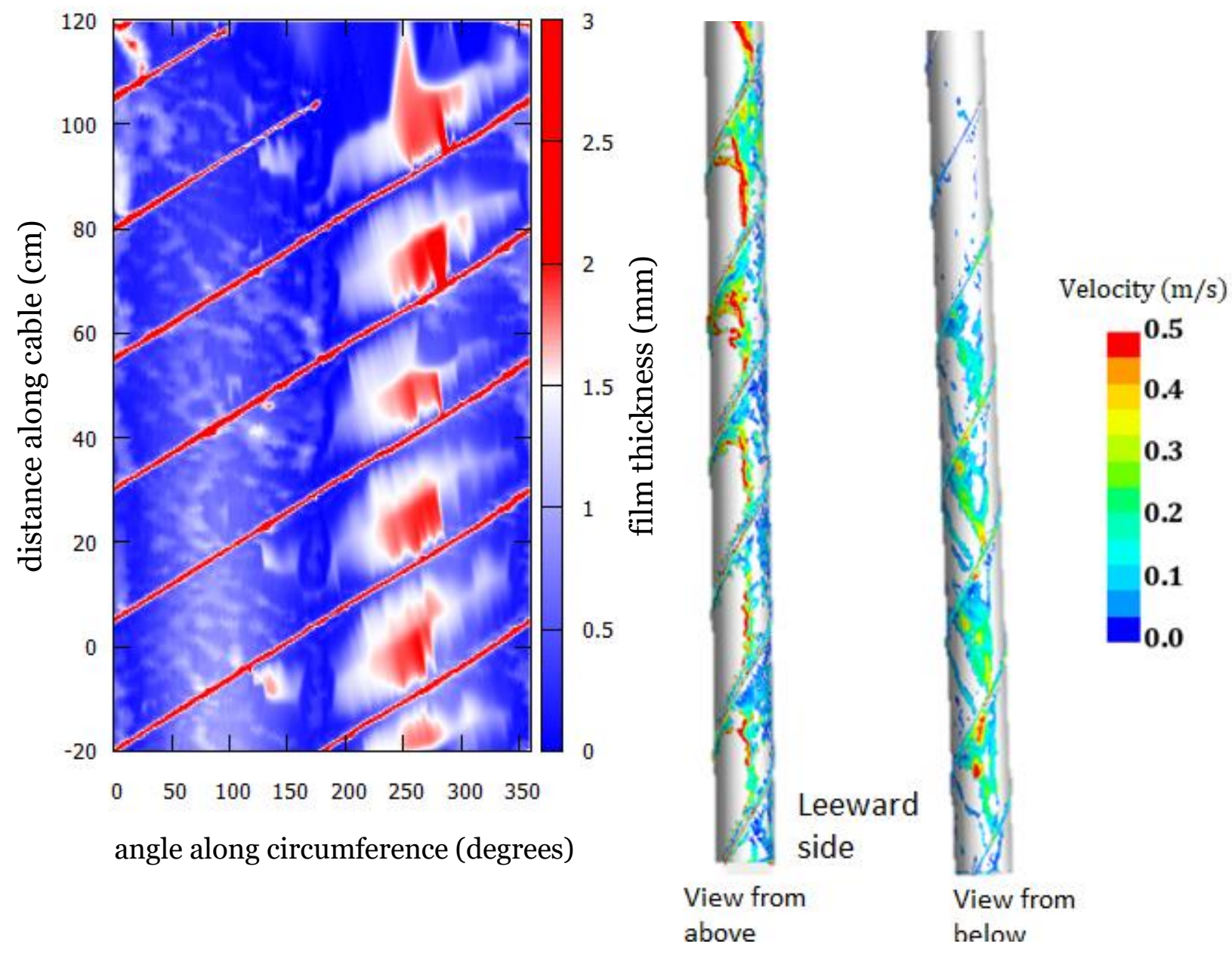

Figure 5-8 Wind velocity $7.2 \mathrm{~m} / \mathrm{s}$ and $60 \mathrm{~mm} / \mathrm{hr}(2.4 \mathrm{in} / \mathrm{hr}$ ) rain: fluid film thickness on the cable surface (left), and film velocity (right). The vertical axis is the length along the axis of the cylinder while the bottom axis is the angle along the circumference of the cylinder with angle 270 deg at the top of the cable. 

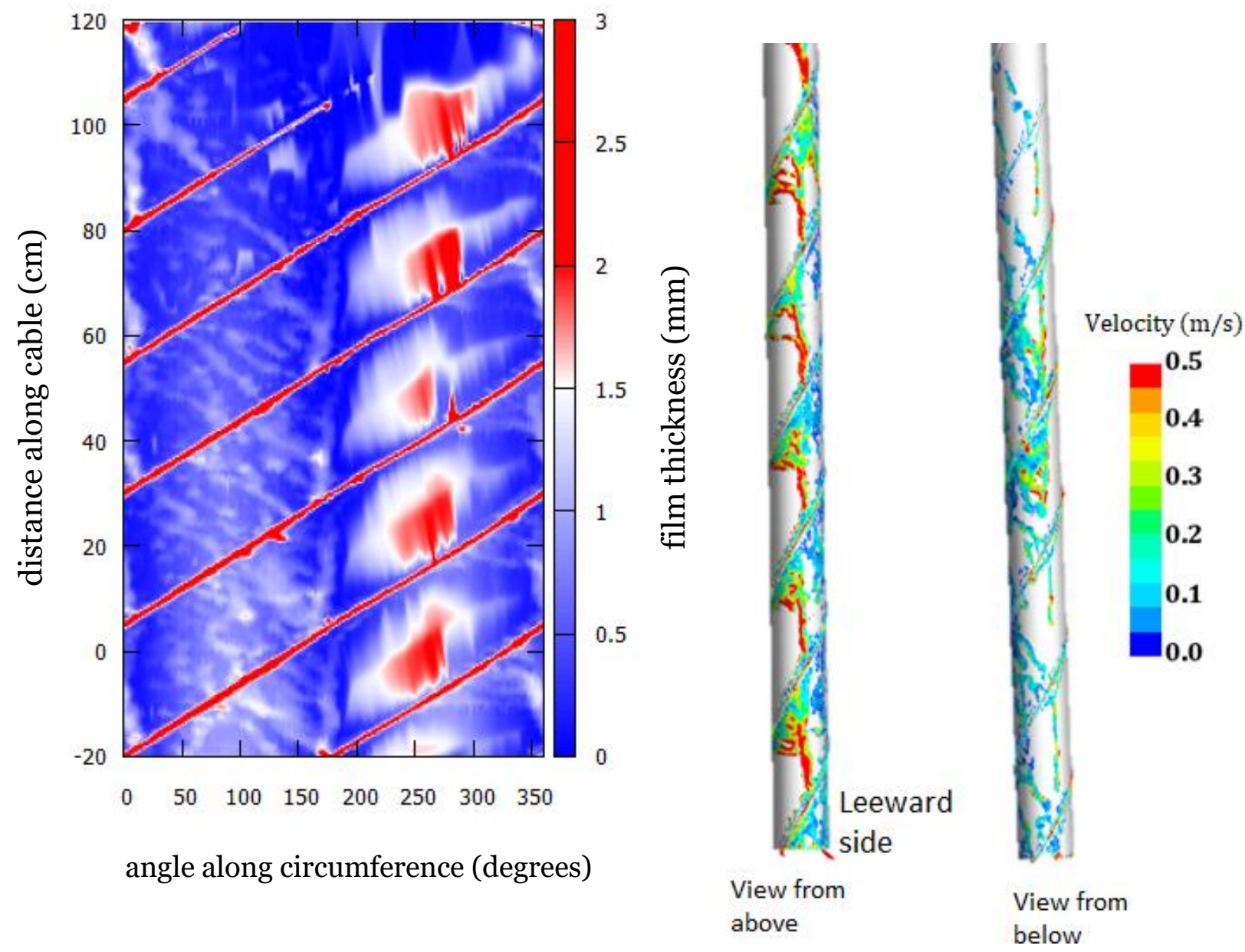

Figure 5-9 Wind velocity $7.7 \mathrm{~m} / \mathrm{s}$ and $60 \mathrm{~mm} / \mathrm{hr}(2.4 \mathrm{in} / \mathrm{hr})$ rain: fluid film thickness on the cable surface (left), and film velocity (right). The vertical axis is the length along the axis of the cylinder while the bottom axis is the angle along the circumference of the cylinder with angle 270 deg at the top of the cable. 

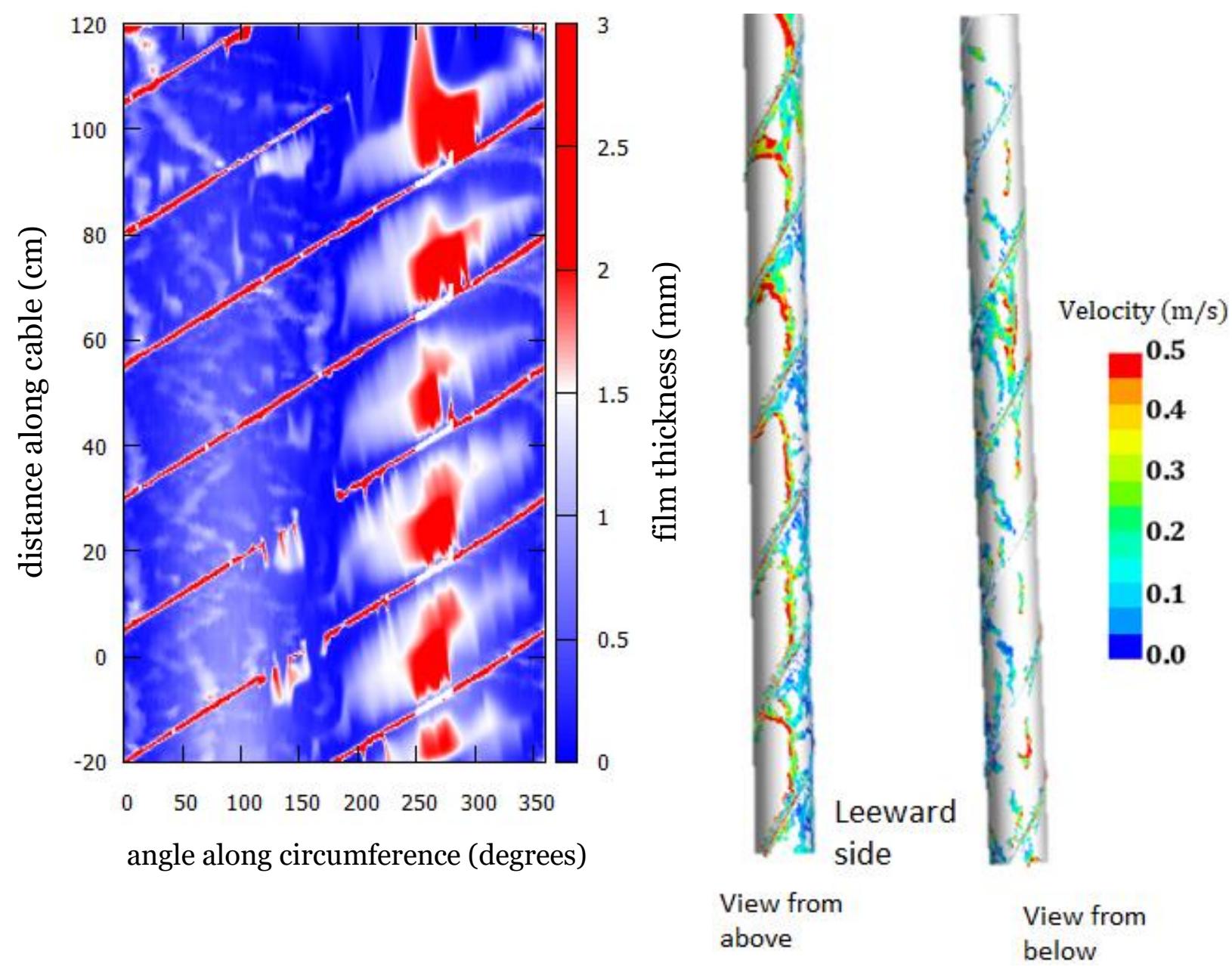

Figure 5-10 Wind velocity $8.7 \mathrm{~m} / \mathrm{s}$ and $60 \mathrm{~mm} / \mathrm{hr}(2.4 \mathrm{in} / \mathrm{hr}$ ) rain: fluid film thickness on the cable surface (left), and film velocity (right). The vertical axis is the length along the axis of the cylinder while the bottom axis is the angle along the circumference of the cylinder with angle 270 deg at the top of the cable. 


\section{Conclusions}

In stay cable bridges, rain-wind induced vibrations can induce excessive stress and could pose a risk for the safety and integrity of the structures. The formation of rivulets on the cable surface, which affects the three-dimensionality of the Karman vortex street, is an important part of the rain-wind induced vibration process. The capability of CFD software to model rivulet formation and flow provides a means to study rivulet distribution and evolution in the presence of fillets that may be used to investigate options to mitigate or reduce the risk of large scale wind-rain induced cable vibrations. This study investigated current advanced multiphase modeling options that can be run efficiently on commonly available mid-range parallel computer clusters using up to a few hundred cores in the 3 D geometry of a stay cable segment with and without fillets.

A fully coupled $3 \mathrm{D}$ modeling technique was developed to study water film formation and the morphology of rivulets flowing under gravity with wind interaction on the cylindrical surface of a stay cable segment. A modeling approach based on a combination of the gas-liquid two-phase theory and the volume of fluid (VOF) technique for modeling free surface flows was found to be a good option for modeling rivulets on stay cable segments. The conditions necessary for the upper and lower rivulets to form

and their flow evolution on the cable surface were studied using the CFD software Star-CCM+. These models appear to be adequate for determining rivulet formation, thickness, and flow on a cable surface with and without fillets.

CFD multiphase physics interaction models exist within STAR-CCM+ and other CFD software to model rain drops with a size spectrum falling on a cable segment along with the water accumulation on the cable surface to form and add to water rivulets on the cable. Computer resources needed to run a model this detailed, however, begin to become prohibitive, extending from days into weeks on a mid-scale parallel computer cluster. An alternative way to model the addition of water at the cable surface is to add source terms for water mass and momentum to the differential equations that add water at the modeled rain intensity and fall velocity. This option for modeling the rain source of incoming water in rivulets has negligible additional computational cost and appears to function well in models focused on the formation and flow of rivulets on a cable surface.

The vortex shedding effects may produce significant lateral (across-wind) vibrations with nonlinear redistributions of cable forces and displacements as well as introduce additional deformations to the cable system. Large eddy simulation can be employed with reasonable run times of 1 to several days on mid-scale computer clusters, 128 to 256 cores or more if available, to capture and study vortex shedding and its effect on the drag coefficient and time varying forces on a cable segment.

CFD analysis without the crosswind showed for a cable segment without fillets that water droplets accumulate in a liquid film forming rivulets that flow toward and along the underside of the cable under the force of gravity. When fillets are present, they intercept and channel the rivulet along the fillet until the fillet is overtopped. This computational result is in agreement with experimental 
observations. In the case of a cable with fillets and crosswind, two distinct symmetrically placed rivulets are formed at the separation zones of the airflow around the cable, which is in agreement with the experiment and previous published numerical results. The thickness of the upper rivulet increases with the increase in wind velocity from $5 \mathrm{~m} / \mathrm{s}$ to $7.7 \mathrm{~m} / \mathrm{sec}$. Also, sub-rivulets are found on the cable due to the Rayleigh-Taylor instability.

\section{Acknowledgements}

The funding for this project came from the Hydraulics Research Program at the Turner-Fairbank Highway Research Center, through Interagency Agreement Number DTFH61-14-X-300002 between DOT and DOE, and the work is performed under DOE's contract with UChicago Argonne, LLC, contract no. DE-ACo2-06-CH11357.

\section{References}

1. Simcenter STAR-CCM+(2018). STAR-CCM+13.04 User Guide. Melville, NY, USA: Siemens AG.

2. Hikami, Y., \& Shiraishi, N. (1988). Rain-wind induced vibrations of cables stayed bridges. Journal of Wind Engineering and Industrial Aerodynamics, 29(1-3), 409-418.

3. Matsumoto, M., Shiraishi, N., \& Shirato, H. (1992). Rain-wind induced vibration of cables and cable-stayed bridges. Journal of Wind Engineering and Industrial Aerodynamics, 4144(1992), 2011-2022.

4. Yamaguchi, H. (1990). Analytical study on growth mechanism of rain vibration of cables. Journal of Wind Engineering and Industrial Aerodynamics, 33(1-2), 73-80.

5. Kleissl, K., \& Georgakis, C. (2012). Comparison of several innovative bridge cable surface modifications. The Seventh International Colloquium on Bluff Body Aerodynamics and Applications (BBAA7), Shanghai, China, September 2-6, 2012

6. Chen, W.-L., Tang, S.-R., Li, H., \& Hu, H. (2013). Influence of Dynamic Properties and Position of Rivulet on Rain-Wind-Induced Vibration of Stay Cables. Journal of Bridge Engineering, 18(10), 1021-1031.

7. Li, S., \& Gu, M. (2006). Numerical Simulations of Flow around Stay Cables with and without Fixed Artificial Rivulets. In Fourth International Symposium on Computer Wind Engineering (pp. 307-310).

8. Rocchi, D., \& Zasso, A. (2002). Vortex shedding from a circular cylinder in a smooth and wired configuration: Comparison between 3D LES simulation and experimental analysis. Journal of Wind Engineering and Industrial Aerodynamics, 9o(4-5), 475-489.

9. $\quad$ Bi, J. H., Wang, J., Lu, P., \& Bao, C. (2012). Numerical Simulation on Rivulets around Stay Cable Subject to Wind. Applied Mechanics and Materials, 256-259, 2700-2703.

10. Bi, J. H., Lu, P., Wang, J., Bao, C., \& Guan, J. (2014). Numerical simulation and analysis of the effects of water-film morphological changes on the aerodynamic lift of stay cables. Journal of Fluids and Structures, 48, 376-392.

11. Rodi, W. (1991). Experience with two-layer models combining the k-epsilon model with a one-equation model near the wall. In 29th Aerospace Sciences Meeting. Reston, Virigina: 
American Institute of Aeronautics and Astronautics.

12. Shih, T., Zhu, J., \& Lumley, J. (1994). Modeling of wall-bounded complex flows and free shear flows. NASA STI/Recon Technical Report NASA-TM-106513.

13. Nikitin, N. V, Nicoud, F., Wasistho, B., Squires, K. D., \& Spalart, P. R. (2000). An approach to wall modeling in large-eddy simulations. Physics of Fluids, 12(7), 1629-1632.

14. Larose, G. L., \& D'Auteuil, A. (2014). Wind Tunnel Investigations of an Inclined Stay Cable with a Helical Fillet.

15. Matsui, T. (1963). Mean velocity distribution in the outer layer of a turbulent boundary layer. Research Report No. 42.

16. Hanjalić, K., \& Launder, B. E. (1972). A Reynolds stress model of turbulence and its application to thin shear flows. Journal of Fluid Mechanics, 52(4), 609-638.

17. Li, F. C., Chen, W. L., Li, H., \& Zhang, R. (2010). An ultrasonic transmission thickness measurement system for study of water rivulets characteristics of stay cables suffering from wind-rain-induced vibration. Sensors and Actuators, A: Physical, 159(1), 12-23.

18. Lemaitre, C., de Langre, E., \& Hémon, P. (2010). Rainwater rivulets running on a stay cable subject to wind. European Journal of Mechanics - B/Fluids, 29(4), 251-258.

19. Taylor, I. J., \& Robertson, A. C. (2015). Numerical investigation of the coupled interaction between an unsteady aerodynamic flow field and a water film coating on a circular cylinder. Journal of Fluids and Structures, 54, 312-331.

20. Mittal, R., \& Balachandar, S. (1995). Effect of three-dimensionality on the lift and drag of nominally two-dimensional cylinders. Physics of Fluids, $7(8), 1841-1865$.

21. Nakayama, Y. (Yasuki), \& Boucher, R. F. (Robert F. . (1999). Introduction to fluid mechanics. Butterworth Heinemann.

22. Bushnell, D., \& Hefner, J. (2000). Viscous Drag Reduction in Boundary Layers. American Institute of Aeronautics and Astronautics.

23. Matteoni, G., \& Georgakis, C. (2012). Aerodynamic force coefficients of plain bridge cables in wet conditions. The Seventh International Colloquium on Bluff Body Aerodynamics and Applications (BBAA7), Shanghai, China, September 2-6, 2012 


\section{Argonne}

Nuclear Engineering and Science Division

Argonne National Laboratory

9700 South Cass Avenue

Argonne, IL 60439-4815

www.anl.gov

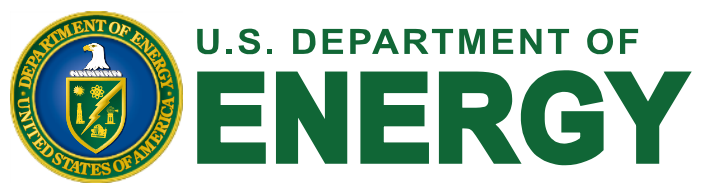

Argonne National Laboratory is a U.S. Department of Energy

laboratory managed by UChicago Argonne, LLC

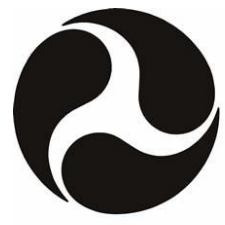

U.S. Department

of Transportation

Federal Highway

Administration 